\title{
Time-varying Formation Dynamics Modeling and Constrained Trajectory Optimization of Multi- quadrotor UAVs
}

\section{Xi Li}

Tiangong University

Guoyuan Qi ( $\sim$ guoyuanqisa@qq.com )

Tiangong University https://orcid.org/0000-0002-0059-4879

Limin Zhang

Zhongyuan University of Technology

\section{Research Article}

Keywords: time-varying, multiple quadrotor UAVs, constraints, optimization

Posted Date: March 11th, 2021

DOl: https://doi.org/10.21203/rs.3.rs-259339/v1

License: (1) (i) This work is licensed under a Creative Commons Attribution 4.0 International License.

Read Full License

Version of Record: A version of this preprint was published at Nonlinear Dynamics on November 2nd, 2021. See the published version at https://doi.org/10.1007/s11071-021-06788-3. 


\title{
Time-varying formation dynamics modeling and constrained
} trajectory optimization of multi-quadrotor UAVs

\author{
Xi Li ${ }^{1}$, Guoyuan $\mathrm{Qi}^{2 *}$, Limin Zhang ${ }^{3}$
}

\section{School of Mechanical Engineering, Tiangong University, Tianjin 300387, China}

2. Tianjin Key Laboratory of Advanced Technology of Electrical Engineering and Energy, Tiangong University, Tianjin 300387, China

3. Department of Electronic and Information Engineering, Zhongyuan University of Technology, Zhengzhou City, Henan Province, 451191, China

Abstract: The formation of multiple quadrotor UAVs with long navigation will encounter many flight constraints, so it is necessary to change the formation to avoid these constraints. In this paper, Voronoi graph theory is used to treat UAVs formation as a rigid body. The rigid body structure can be changed by changing the formation before different constraints, after passing constraints the rigid body structure remains the formation unchanged or form into the prescribed formation. The timevarying model is established to facilitate the use of optimization. Based on Gauss pseudospectral method (GPM), the path optimization of a single quadrotor UAV is carried out. The followed UAVs in formation are treated as constraints. The constraints of maximum turning radius and formation transformation time of other UAVs are considered in the optimization process. The minimum time required for formation transformation is optimized to solve the transformation optimization, and the performance index of trajectory optimization is to minimize the energy consumed by the leader quadrotor UAV within the specified time. Finally, the simulation proves the method proposed in this paper.

Key Words: time-varying, multiple quadrotor UAVs, constraints, optimization

\section{Introduction}

The cooperative path planning of multi-UAVs is the crucial technology of UAVs cooperative combat mission planning system [1-5]. The constraints of multi-UAVs collaborative path planning not only consider the physical performance and the mission requirements of a single UAV, but also the cooperative relationship among all UAVs is taken into account, which includes achieving the 
target constraint at the same time and the minimum safe distance constraint between multiple UAVs [6-8]. When the environment changes, the formation of multi-UAVs should be adjusted in real-time according to the environment to ensure the survival of UAVs $[9,10]$.

In the past, the research on the path planning of multiple UAVs mainly have focused on the assembling of multiple UAVs and the respective path planning of multiple UAVs, while the path planning of multiple UAVs maintaining formation has been studied less. Turpin et al. [11] considered the problem of controlling a group of Mavs to move rapidly in a 3D environment while maintaining a tight formation. The flight trajectory of a certain UAV was parameterized by using the coefficients of sequence polynomial function, and the flight trajectory is fully described according to these values. At the same time, in literature [12], each UAV independently plans its trajectory based on the local information of its neighboring robots, including the planned trajectory of its neighbors and the estimation of its state. In literature [13], aiming at multiple UAVs arrived at the target position from different positions, Shanmugavel et al. proposed a three-stage collaborative path planning method using Dubins paths with clothoid arcs when the constraints were met. Nikolos et al. [14] used an improved genetic algorithm to realize the 2-D trajectories planning of multiple UAVs. Besada-Portas et al. [15] adopted a centralized path planning algorithm based on the genetic algorithm to plan the path for multiple UAVs. Radmanesh et al. [16] researched the collision-free 3D trajectories for multiUAVs in shared airspace, and proposed a decentralized method based on a Partial Differential Equation, in which, the dynamic characteristics of multiphase fluids in porous media are utilized, and the porosity is modeled as a function of collision risk. Chen et al. [17] solved the formation control path planning problem of multiple quadrotor UAVs by introducing virtual rate rigid body and virtual target point and updating the artificial potential field method based on additional control force.

At present, Gauss pseudospectral method (GPM) has many applications in trajectory optimization. To optimize the takeoff-mass for a two-stage-to-orbit RBCC-RKT launch vehicle, which is a crucial factor of integral performance, Zhang et al. [18] combined it with the trajectory optimization simultaneously by treating them as a nonlinear control problem, when the scopes of initial and terminal qualities as well as speeds are specified, the problem is solved by using the Gauss pseudospectral method. In literature [19], in order to optimize the trajectory of a general supersonic vehicle driven by a hydrocarbon fueled ramjet in the ascent phase, an optimization method based on GPM was proposed based on the characteristics of the propulsion system. In literature [20], in order 
to solve the problem of autonomous landing trajectory optimization of mobile platform, with the minimum of time and energy consumption as the optimal goal, the GPM method was used to optimize the landing trajectory of a tilt rotor. However, there have been few literatures studied on the path optimization of variable formation of the quadrotor UAVs formation under the constraint of threedimensional space.

To the best of our knowledge, there is no report about the conversion of the information of followed quadrotor UAVs(QUAVs) in formation into the constraints of the leader QUAV. Research on the information transformation of followed QUAVs in QUAVs formation into the constraints of leader QUAV can make the formation trajectory optimization problem stand-alone, simplify the complex multi-QUAVs formation trajectory optimization problem, and ensure the maintenance of multi-QUAVs formation and the timeliness of cooperation, and can optimize multiple performance indexes at the same time.

In this paper, we propose the path optimization problem of QUAVs formation with obstacles in three-dimensional space through kinematics analysis and numerical simulation. The QUAVs formation is regarded as a virtual rigid body, and the rigid body structure can be changed by changing the formation before different constraints. After pass one constraint, the QUAVs formation remains unchanged or changes to the specified formation. And the optimization model is established in this paper, and the optimization improvement of the end heading constraint and height constraint is proposed. The optimization model is transformed into a sequential quadratic programming problem by Gauss pseudospectral method.

The rest of this article is organized as follows. Section 2 introduces the model of a QUAV attitude system, and the dynamic model of multiple QUAVs is established to analyze the constraints and formation maintenance during its flight. Finally, the problems summary faced in the flight trajectory optimization model for QUAVs formation is given. In Section 3, principle of Gauss pseudospectral method is introduced and used to solve the problems in Section 2. In Section 4, simulation examples are given. Section 5 draws some conclusions.

\section{Dynamics model of multiple QUAV}

\subsection{Dynamics model of a QUAV}


A QUAV is a kind of aircraft with four rotors, having two types of X-type distribution and crosstype distribution $[21,22]$. In this paper, the X-type is used, and the schematic diagram of the QUAV is shown in Fig. $1[23,24]$.

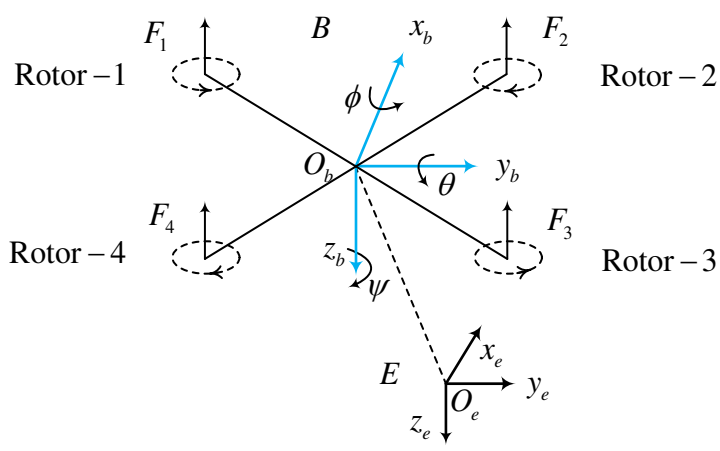

Fig. 1 Schematic view of the QUAV system.

The quadrotor UAV can achieve various motions by changing the rotor speed. In the figure, $\{E\}=\left\{x_{e}, y_{e}, z_{e}\right\}$ is the north-east-down inertial coordinate system (NED). $\{B\}=\left\{x_{b}, y_{b}, z_{b}\right\}$ is the body coordinate system, the origin of which coincides with the mass center of the body, and the coordinate axis is fixedly connected with the QUAV. The x-axis is the forward direction of the UAV. $F_{i}(t), i=1,2,3,4$ is the thrust produced by the four motors, respectively. $\{x(t), y(t), z(t)\}$ is the position of the QUAV in the local NED frame $\{E\}$, and $\{\phi(t), \theta(t), \psi(t)\}$ is the Euler angle of the QUAV.

To establish a relatively accurate mathematical model of QUAV, the following assumptions are made:

(1) The small quadrotor UAV is regarded as a rigid body and is completely symmetrical;

(2) The origin of body coordinate $O_{b}$ is completely coincident with the mass center of UAV;

(3) The propeller of UAV is a rigid-body without considering its structure and elastic deformation.

The nonlinear dynamic model of the QUAV in $\{E\}$ is given as follows $[25,26]$ : 


$$
\left\{\begin{array}{l}
\ddot{x}=-(\cos \psi \sin \theta \cos \phi+\sin \psi \sin \phi) \frac{U_{1}}{m}+\frac{K_{1} \dot{x}}{m}, \\
\ddot{y}=-(\sin \psi \sin \theta \cos \phi-\cos \psi \sin \phi) \frac{U_{1}}{m}+\frac{K_{2} \dot{y}}{m} \\
\ddot{z}=-(\cos \theta \cos \phi) \frac{U_{1}}{m}+g+\frac{K_{3} \dot{z}}{m}, \\
\ddot{\phi}=\frac{1}{I_{x}}\left[l U_{2}+\dot{\theta} \dot{\psi}\left(I_{y}-I_{z}\right)-K_{4} \dot{\phi}\right] \\
\ddot{\theta}=\frac{1}{I_{y}}\left[l U_{3}+\dot{\phi} \dot{\psi}\left(I_{z}-I_{x}\right)-K_{5} \dot{\theta}\right], \\
\ddot{\psi}=\frac{1}{I_{z}}\left[U_{4}+\dot{\theta} \dot{\phi}\left(I_{x}-I_{y}\right)-K_{6} \dot{\psi}\right],
\end{array}\right.
$$

where, $x, y, z$ are the positions of the QUAV; $I_{x}, I_{y}, I_{z}$ are the rotational inertias of the QUAV respectively; because the mechanical structure of the QUAV is completely uniform, so $I_{x}=I_{y}$; $\phi, \theta, \psi$ are the roll angle, pitch angle and yaw angle of the QUAV respectively; in the inertial frame of reference, $\dot{\phi}$ is roll angle velocity, $\dot{\theta}$ is pitching angle velocity, and $\dot{\psi}$ is yaw angle velocity; $l$ is the length between the center of the aircraft and the rotor; $U_{1}$ is the vertical motion control quantity, $U_{2}$ is the rolling motion control quantity, $U_{3}$ is the pitch motion control quantity, $U_{4}$ is the yaw motion control quantity. $K_{1}, K_{2}, K_{3}$ are the coefficient of air resistance, $K_{4}, K_{5}, K_{6}$ represent the coefficient of air resistance moment. In addition, $\left[v_{x}, v_{y}, v_{z}\right]$ and $\left[w_{\phi}, w_{\theta}, w_{\psi}\right]$ are defined as linear velocity components and angular velocity components around $x, y, z$ coordinate in the NED coordinate system, where $v_{x}$ is the forward velocity, $v_{y}$ is the lateral velocity, $v_{z}$ is the falling velocity, $w_{\phi}$ is the roll angular velocity, $w_{\theta}$ is the pitch angular velocity, and $w_{\psi}$ is the yaw angle angular velocity.

The expression of the control quantity is expressed as follows: the control quantity directly controls the motor speed, and the actual implementation form is PWM wave to control the motor.

$$
\left[\begin{array}{c}
U_{1} \\
U_{2} \\
U_{3} \\
U_{4}
\end{array}\right]=\left[\begin{array}{c}
K_{T}\left(\Omega_{1}^{2}+\Omega_{2}^{2}+\Omega_{3}^{2}+\Omega_{4}^{2}\right) \\
\frac{\sqrt{2}}{2} K_{T}\left(\Omega_{1}^{2}-\Omega_{2}^{2}-\Omega_{3}^{2}+\Omega_{4}^{2}\right) \\
\frac{\sqrt{2}}{2} K_{T}\left(\Omega_{1}^{2}+\Omega_{2}^{2}-\Omega_{3}^{2}-\Omega_{4}^{2}\right) \\
K_{M}\left(\Omega_{1}^{2}-\Omega_{2}^{2}+\Omega_{3}^{2}-\Omega_{4}^{2}\right)
\end{array}\right],
$$


where, $\Omega_{i}(i=1,2,3,4)$ represents the rotation speed of each propeller, $K_{T}$ represents the propeller lift coefficient, $K_{M}$ represents the inverse torque coefficient of the propeller.

\subsection{Description of flight problems of multiple QUAVs}

The formation of QUAVs is shown in Fig. 2.

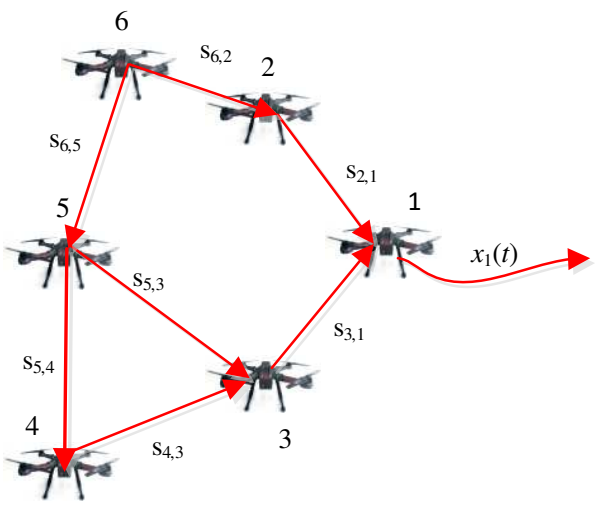

Fig. 2 Formation of QUAVs

The formation of QUAVs follow the leader (UAV1) which tracks the desired trajectory $x_{1}(t)$.

Each UAV is controlled to maintain a desired shape defined by shape vectors and denoted by $s_{i, j}$. In this paper, the formation is composed of $N$ QUAVs. Due to turning or other situations when flying in formation, if multiple QUAVs keep the formation unchanged, their speeds may be inconsistent. Therefore, the following assumptions are defined in this paper:

(1) The speed of the leader UAV remains unchanged during flight. When it is not turning, all UAVs move at a constant speed. When it is flying in formation, the speed of each UAV is not consistent. It doesn't take into account the energy consumption of the turn.

(2) The UAVs can communicate reliably to prevent collisions among them.

(3) The UAVs formation can be changed according to the environmental constraints. After passing the obstacle, the formation recovers and continues to fly at the same speed and direction, or continues to fly with the formation unchanged.

(4) The formation of QUAVs is regarded as a virtual rigid body. Except for formation transformation, the formation of UAVs is regarded as a rigid body.

Virtual structure method: The basic idea of the virtual structure method is to regard the UAV formation system as a virtual structure of a rigid-body. Each UAV is considered as a fixed point on the virtual structure. When the UAVs' formation moves, the UAV can follow the corresponding fixed 
point on the rigid body to achieve fixed formation flight.

In this paper, the consistency theory based on Voronoi graph theory [27-29] is used to define the formation keeping problem among the QUAVs. The formation system is transformed into a network topology. The state information of UAVs is consistent with the expected information through the consistency algorithm to realize the autonomous formation movement control of multiple UAVs.

According to the information communication relationship among the formation formed by $N$ QUAVs, the flight formation is modeled as a directed graph $G$. The directed graph node $v_{i}$ represents the $i$-th QUAV. The direction of information flow is represented by the direction of arrows in the directed graph $G$. The node $v_{1}$ represents the leader UAV, the remaining nodes represent the followed UAVs $v_{i}(t)=\left[x_{i}(t), y_{i}(t), z_{i}(t), \psi_{i}(t), \theta_{i}(t), \phi_{i}(t)\right]^{\mathrm{T}}$. In the leader-followed formation mode, the leader position is the equilibrium point of the formation consistency algorithm. The expected position deviation between the leader UAV and followed UAVs is defined as the relative position deviation. The leader UAV sends its state to the followed UAVs. The topology of information among the followed UAVs can be any directed graph. The shape vectors are $6 \times 1$ vectors relating positions of pairs of UAVs. For UAV $i$ and $j$ :

$$
v_{i, j}(t)=v_{j}(t)-v_{i}(t)=\left[\begin{array}{c}
x_{j}(t)-x_{i}(t) \\
y_{j}(t)-y_{i}(t) \\
z_{j}(t)-z_{i}(t) \\
\psi_{j}(t)-\psi_{i}(t) \\
\theta_{j}(t)-\theta_{i}(t) \\
\phi_{j}(t)-\phi_{i}(t)
\end{array}\right] .
$$

These will be used to describe the desired relative configuration for each pair of UAVs in Fig. 2. In the rest of the paper, we assume UAV1 is specified as the leader for the team and the group motion, $g(t)=x_{1}$. The overall shape can be prescribed by a $(N-1) \times(N-1)$ vector-valued, skewsymmetric matrix [27].

Considering the change of the communication topology among the formation systems of the QUAVs with the time change, let $G=\left\{G_{1}, G_{2}, \cdots, G_{m}\right\}, m \geq 1$ represents the possible formation set, and $m$ represents the maximum number of possible formations.

Definition 1 [30]: Time-varying formation can be represented by a set of functions 
$h_{i}(t), i=1,2, \cdots, N$, for a given formation $h_{i}(t)$ and a certain trajectory $c(t)$, if the formation system of UAVs satisfies in any given initial situation

$$
\lim _{t \rightarrow \infty}\left(\xi_{i}(t)-h_{i}(t)-c(t)\right)=0, i=1,2, \cdots, N
$$

then it is said that the UAVs achieve formation $h_{i}(t) . c(t)$ is called the center track of formation. where, $\xi_{i}(t)=\left[x_{i}(t), v_{i}(t)\right]^{\mathrm{T}}$, here $x_{i}(t)$ and $v_{i}(t)$ respectively represent the position and velocity in the three-dimensional space of the $i$-th UAV.

When a UAV formation encounters an obstacle, it sometimes needs to change its formation to get through the obstacle. Formation transformation in the flight of UAVs formation can be regarded as reassembly of UAVs, namely formation reconstruction. At this time, the UAVs formation is regrouped from formation A into new formation B, as shown in Fig.3. In formation transformation, minimum energy consumption or time is required [31]. In this paper, the minimum time is taken as the required target of formation transformation.

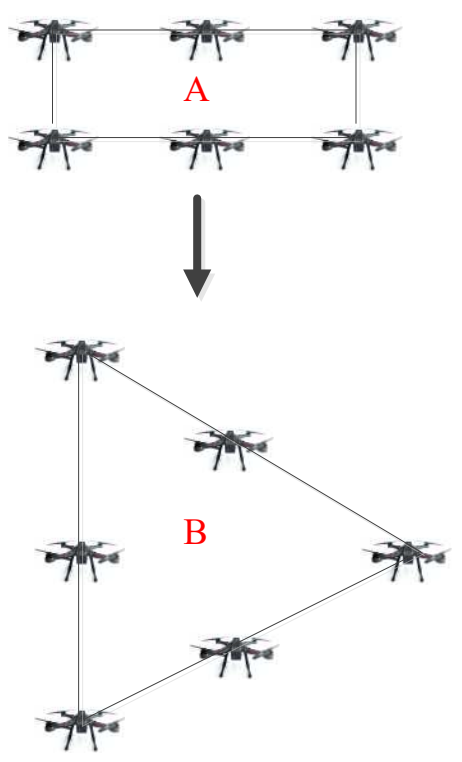

Fig.3 Formation transformation

QUAVs have the advantages of hovering and maneuvering in all directions, so formation change can be made by methods of formation rotation, formation reduction or new formation.

(1) Rotating

To keep the formation rigid, the formation can be rotated at the same angle around a centerline or a center point to keep the relative position of each UAV unchanged. Supposed the position of the $i$-th UAV in the inertial coordinate system is $\left(x_{i}, y_{i}, z_{i}\right)$, the position of its rotation center in the 
inertial coordinate system is $\left(x_{r}, y_{r}, z_{r}\right)$, and the rotation angles in three-dimensional space are $\alpha$, then the rotation path length of the $i$-th UAV is an arc, and the arc length is

$$
l_{i}=\alpha\left[\left(x_{i}-x_{r}\right)^{2}+\left(y_{i}-y_{r}\right)^{2}+\left(z_{i}-z_{r}\right)^{2}\right]^{1 / 2} .
$$

If the minimum rotation time $t$ is required, only the minimum rotation time corresponding to the UAV with the maximum rotation length $l_{\max }$ is required, namely, let Eq.(5) be minimized.

$$
J_{1}=t_{\text {min }}=\min \left(\max \left(\frac{l_{i}}{v_{i}}\right)\right), i=1,2, \cdots, N
$$

At this time, the rotation speed of other UAVs is

$$
v_{i}=\frac{l_{i}}{t_{\min }}, i=1,2, \cdots, N .
$$

If the UAV with the maximum rotation path is allowed to reach its maximum flight speed $v_{\max }$, it can also be obtained

$$
t_{\min }=\frac{l_{\max }}{v_{\max }}
$$

All UAVs can rotate synchronously.

After passing the obstacle, then the formation rotates back to the original formation at an angle of $-\alpha$ and continues to fly, or keep the formation unchanged.

(2) Formation shrinking

Formation of QUAVs draw close to the center of formation in proportion to shrink formation and pass obstacles, as shown in Fig. 4 [31].

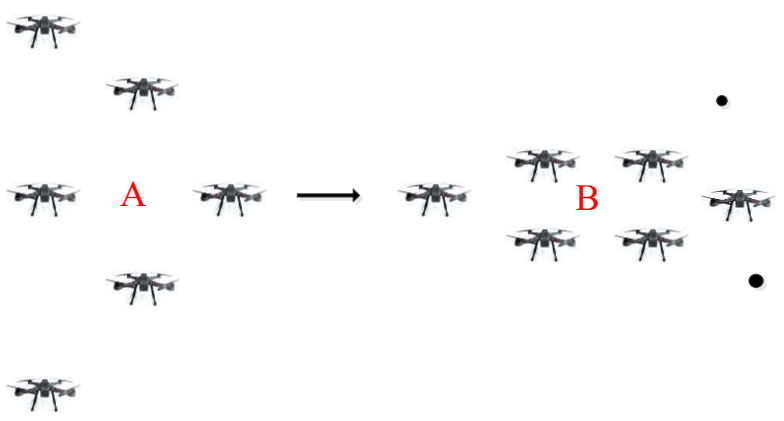

Fig. 4 Reduction diagram of formation

Formation shrinking can be regarded as a translational movement. In this paper, formation shrinking transformation is still carried out based on the requirement of minimum time. The distance of the UAV farthest from the center $f\left(x_{f}, y_{f}, z_{f}\right)$ and formation center $\left(x_{r}, y_{r}, z_{r}\right)$ is 


$$
l_{\text {max }}=\left[\left(x_{f}-x_{r}\right)^{2}+\left(y_{f}-y_{r}\right)^{2}+\left(z_{f}-z_{r}\right)^{2}\right]^{1 / 2} .
$$

212

213

If the formation shrinking time $t$ is required to be the minimum, then only the transformation time corresponding to UAV $f$ is required to be the minimum, namely

$$
J_{2}=t_{\min }=\min \left(\frac{l_{f}}{v_{f}}\right) .
$$

At this time, the rotation speed of other UAVs is

$$
v_{i}=\frac{l_{i}}{t_{\min }}, i=1,2, \cdots, N .
$$

If the UAV is allowed to fly at its maximum speed $v_{\max }$, we can also get

$$
t_{\min }=\frac{l_{\max }}{v_{\max }} .
$$

That is, all UAVs can rotate synchronously.

After passing the obstacle, the formation recovers and continues to fly at the same speed and direction, or continues to fly with the formation unchanged.

(3) The new formation

When the UAVs meet some constraints and need to be transformed into a new formation, the UAV formation can be regarded as regrouping without obstacles. The minimum time is required as the performance index requirements. Here, if all UAVs are required to fly at a constant speed $v_{\max }$ with their maximum speed, the time $t_{i}$ taken by each UAV is proportional to its distance, namely,

$$
t_{i}=\frac{l_{i}}{v_{\max }} .
$$

It can be based on the existing formation regrouping methods (such as literature [2, 6, 7, 31], etc.), taking the following as the objective function to solve the optimal value:

$$
J_{3}=t_{\min }=\min \left\{\max t_{i}\right\}, i=1,2, \cdots, N .
$$

After $t_{\min }$ is obtained, the UAVs can fly at a constant speed $v_{i}=l_{i} / t_{\min }$ again to realize synchronous formation transformation.

\subsection{The constraints of leader UAV}


(1) Initial boundary condition constraint

The initial condition means that the UAV needs to meet the initial position and attitude constraints when at the initial point $t=0$, namely:

$$
X\left(t_{0}\right)=X_{0}, Y\left(t_{0}\right)=Y_{0}, Z\left(t_{0}\right)=Z_{0}, \theta\left(t_{0}\right)=\theta_{0}, \phi\left(t_{0}\right)=\phi_{0}, \psi\left(t_{0}\right)=\psi_{0} .
$$

(2) Terminal condition constraint

The terminal condition refers to the terminal position and terminal attitude constraints of the state variable that need to be met when the UAV arrives at the target point at $t=t_{f}$, namely:

$$
X\left(t_{f}\right)=X_{f}, Y\left(t_{f}\right)=Y_{f}, Z\left(t_{f}\right)=Z_{f}, \theta\left(t_{f}\right)=\theta_{f}, \phi\left(t_{f}\right)=\phi_{f}, \psi\left(t_{f}\right)=\psi_{f} .
$$

(3) Minimum turning radius

Because the flight physics of the UAV determines the value of its turning radius, the limitation of the turning radius should be taken into account when the path planning is carried out; otherwise, the UAV cannot fly according to the set path. Besides, a larger turning radius prevents the UAVs from colliding with each other when they change formation. The minimum turning radius refers to the circular radius of the UAV when it moves in a circular motion with extreme acceleration at the horizontal plane. In general, this radius should meet the following requirements:

$$
\frac{V_{1}^{2}}{R_{1} \cdot g} \leq n_{1 \max }
$$

where, $V_{1}$ is the speed of the leader UAV, $R_{1}$ is the turning radius of the leader UAV, $g$ is the gravitational acceleration, and $n_{1 \max }$ is the maximum normal acceleration of the leader UAV. We can obtain the minimum transition radius of the leader UAV flight, which shall meet the following requirements:

$$
R_{1} \geq \frac{V_{1}^{2}}{n_{1 \max } \cdot g}
$$

(4) Control constraints

$$
U_{i \min } \leq U_{i} \leq U_{i \max }, U_{i \max }>0, U_{i \min } \leq 0, i=1,2,3,4 .
$$

(5) Flight altitude minimum constraint

In general, UAV will encounter high buildings, mountains or enemy fire range during flight. If the UAV flies too low, it will increase the probability of collision or be shot down. Therefore, the minimum height of the UAV flight should be set during path planning to ensure that all UAVs fly 
above the safe height.

$$
z_{1}(t) \geq h_{\text {min }}(t), h_{\text {min }}(t)>0,
$$

where, $z_{1}(t)$ is the real-time flying altitude of the leader UAV in the formation, and $h_{\min }(t)$ is the corresponding real-time minimum flying altitude requirement.

(6) Horizontal constraint

When flying in a certain height, it is also needed to avoid some obstacles in horizontal direction, including mountains, islands, generally political no-fly zone, etc. It is needed to keep a safe distance to the peripheral contour of the obstacle to prevent the UAV from colliding with obstacles due to inertia or lack of mobility. Meanwhile, when the obstacle is a building, keeping a certain distance from the obstacle can also reduce the probability of the UAV being detected by the enemy. In this paper, at a certain flying altitude, the horizontal obstacles are represented by discs and the constraints to be satisfied in the horizontal direction are as follows:

$$
\left(x_{1}(t)-x_{j}\right)^{2}+\left(y_{1}(t)-y_{j}\right)^{2}>R_{j}, j=1,2, \cdots, m,
$$

where, $\left(x_{1}(t), y_{1}(t)\right)$ represents the real-time position of the leader UAV, $\left(x_{j}, y_{j}\right)$ and $R_{j}$ represents the center and radius of the $j$ no-fly zone disk respectively, and $m$ represents the total number of no-fly zones.

\subsection{The constraints of followed UAVs}

The leader UAV is only considered that path optimization of multi-QUAVs formation studied in this paper, and the followed UAV exists as a constraint condition. Its conversion into constraints is dealt with as follows:

(1) According to the position information of followed UAVs and leader UAV in formation, the position of followed UAV in the inertial coordinate system can be expressed as follows:

$$
\left\{\begin{array}{l}
x_{i}(t)=x_{1}(t)-x_{i 1}, \\
y_{i}(t)=y_{1}(t)-y_{i 1}, \\
z_{i}(t)=z_{1}(t)-z_{i 1},
\end{array}\right.
$$

where, $\left(x_{i}(t), y_{i}(t), z_{i}(t)\right)$ is the real-time position of the $i$-th followed UAV, $\left(x_{1}(t), y_{1}(t), z_{1}(t)\right)$ is the real-time position of the leader UAV, and $\left(x_{i 1}, y_{i 1}, z_{i 1}\right)$ is the relative 


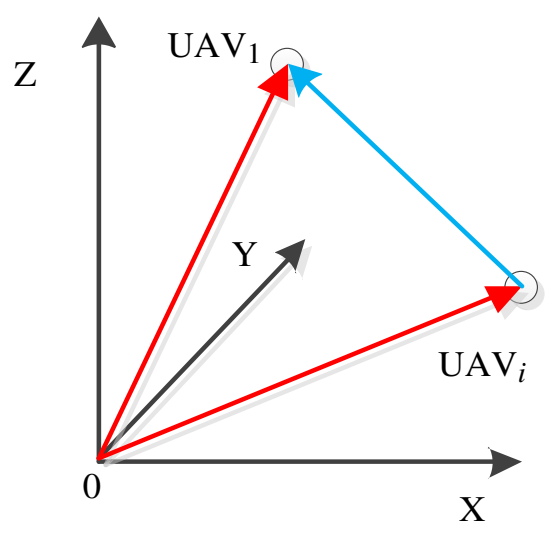

Fig. 5 Relative position in formation between followed UAV and leader UAV

In this case, the followed UAV constraints can be transformed into constraints on the leader UAV.

(2) Flight altitude minimum

$$
\left\{\begin{array}{l}
z_{i}(t)=z_{1}(t)-z_{i 1} \geq h_{\text {min }}(t) \\
\Rightarrow z_{1}(t) \geq h_{\text {min }}(t)+z_{i 1}, \quad h_{\text {min }}(t)>0 .
\end{array}\right.
$$

(3) Horizontal constraint

$$
\begin{aligned}
& \left(x_{i}(t)-x_{j}\right)^{2}+\left(y_{i}(t)-y_{j}\right)^{2} \\
= & \left(x_{1}(t)-x_{i 1}-x_{j}\right)^{2}+\left(y_{1}(t)-y_{i 1}-y_{j}\right)^{2} \\
> & R_{j}, j=1,2, \cdots, m .
\end{aligned}
$$

(4) Turning radius minimum

Like the leader UAV, the turning radius of the followed UAVs should also meet the requirements:

$$
\frac{V_{i}^{2}}{R_{i} \cdot g} \leq n_{i \max }
$$

where, $V_{i}$ is the speed of the $i$-th followed $\mathrm{UAV}, R_{i}$ is the turning radius of the $i$-th followed UAV, $g$ is the gravitational acceleration, and $n_{i \max }$ is the maximum normal acceleration of the $i$-th followed UAV. For this purpose, we obtain a minimum flight transition radius $R_{i}=\frac{V_{i}^{2}}{n_{i \max } \cdot g}$ of the $i$-th followed UAV.

In formation flight, the leader UAV and followed UAVs rotate around the same center, that is, fly around the same center of a circle. The speed has the following relationship: 


$$
\begin{aligned}
& \frac{V_{i}}{V_{1}}=\frac{\frac{1}{2} \pi R_{i}^{2}}{\frac{1}{2} \pi R_{1}^{2}}=\frac{R_{i}^{2}}{R_{1}^{2}} \\
& \Rightarrow V_{i}=\frac{R_{i}^{2}}{R_{1}^{2}} V_{1},
\end{aligned}
$$

According to Fig. 5,

$$
R_{i}=\sqrt{x_{i}^{2}+y_{i}^{2}+z_{i}^{2}}=\sqrt{\left(x_{1}-x_{i 1}\right)^{2}+\left(y_{1}-y_{i 1}\right)^{2}+\left(z_{1}-z_{i 1}\right)^{2}},
$$

We can get

$$
\frac{V_{i}^{2}}{R_{i} \cdot g}=\frac{\left(\frac{R_{i}^{2}}{R_{1}^{2}} V_{1}\right)^{2}}{R_{i} \cdot g}=\frac{R_{i}^{3}}{R_{1}^{4}} V_{1}^{2} .
$$

That is

$$
\frac{\left(\sqrt{\left(x_{1}-x_{i 1}\right)^{2}+\left(y_{1}-y_{i 1}\right)^{2}+\left(z_{1}-z_{i 1}\right)^{2}}\right)^{3}}{R_{1}^{4}} V_{1}^{2} \leq n_{i \max } .
$$

Thus, they can be converted to related the minimum turning radius, speed and position constraints of the leader UAV.

\subsection{Improvement of the performance index function}

(a) Energy consumption index for total flight distance

When formation transformation is not taken into account in formation flight, the minimum energy consumption performance index traditionally adopted is:

$$
J_{4}=\min \left(\sum_{i=1}^{N} \omega_{i}\right)
$$

where, $\omega_{i}=\int_{t_{0}}^{t_{f}} \omega_{i}(t) d t$ denotes the total energy consumption of $\mathrm{UAV}_{i}$, and $\omega_{i}(t)$ denotes the realtime energy consumption. For all $\mathrm{UAV}_{i}$, since the concept of virtual rigid formation is adopted in this paper, and there is a cooperative time constraint, each UAV must reach the specified location at the same time $t_{f}$, so the speed and distance of all $\mathrm{UAV}_{i}$ are in direct proportion [31], namely

$$
v_{i}=\frac{l_{i}}{t_{f}-t_{0}} .
$$

If the impacts of different $\mathrm{UAV}_{i}$ on air density and windward area, as well as thermal effects, etc. are 
ignored, the energy consumption generated by $\mathrm{UAV}_{i}$ is

$$
\omega_{i}=F_{i} l_{i}
$$

where, $F_{i}=k_{f} v_{i}^{2}$ represents the air resistance during $\mathrm{UAV}_{i}$ flight, $k_{f}$ is the air resistance coefficient, and is a constant value. The same UAV is used in this paper, so $k_{f}$ of all UAVs are of the same value.

(b) Analysis on the influence of formation transformation

The formation transformation used in this paper takes the leader UAV as the central axis for transformation. The leader UAV used still follows its trajectory during formation transformation. It's the followed UAVs that make the formation change. Formation change or rotation can be ignored relative to the total flight distance. The formation change can also be completed during the flight. Therefore, the time solution of formation change can be completed online quickly, and the influence on the overall flight optimization of the leader UAV can be ignored.

(c) The terminal demand

Generally speaking, if a UAV formation is required to fly in a straight line from a certain horizontal direction at a certain altitude to the target point, in order to ensure the UAV's terminal flight path is straight and reduce the control demand at the terminal stage, we introduce the following performance index function:

$$
\begin{gathered}
J_{5}=\min \left(Q(t)\left(\psi_{1}(t)-\psi_{r}\right)\right), \\
J_{6}=\min \left(Q(t)\left(z_{1}(t)-h_{r}\right)\right),
\end{gathered}
$$

where, $Q(t)$ is the weighting function. To ensure the requirements of the terminal flight, we finally adopt the performance index function as follows:

$$
J=a_{1} J_{4}+a_{2} J_{5}+a_{3} J_{6}
$$

where, $a_{1}, a_{2}, a_{3}$ are the weighting coefficients, which can be selected according to the actual requirements. In this paper, the energy consumption index of UAV takes up a significant proportion and $a_{1}=0.8, a_{2}=0.1, a_{3}=0.1$.

The flight track diagram and control curve of the improved single QUAV are shown in Fig. 6, in which different weighting functions are added, respectively [32]: 

(1) $Q(t)=1$;
(2) $Q(t)=t^{2}$
(3) $Q(t)=1 /\left(t_{f}-t+0.5\right)$;
(4)
$Q(t)=1 /\left(t_{f}-t+0.5\right)^{2}$

In order to verify the influence of different weighting functions $Q(t)$, the following simulation

352

353 is carried out: a single QUAV takes off from $(0,0,2) m$ and lands on $(1000,1000,2) m$, and the end requires the UAV to fly from $-90^{\circ}$ direction to the target point and gradually land from a higher altitude. Fig. 6 is the $x-y$ plane flight diagram, and Fig. 7 is the corresponding 3D flight diagram.

As can be seen from Fig. 6, the four weighting functions selected by us can ensure that the UAV will turn into a flat state in the latter part of flight. However, as can be seen from the 3D flight diagram in Fig. 7, when taking $Q(t)=1$, the altitude of UAV in the later period of flight will change a lot, and it cannot be guaranteed that the UAV will land from a higher position; when taking $Q(t)=t^{2}$ and $Q(t)=1 /\left(t_{f}-t+0.5\right)^{2}$, the altitude of UAV in the later period of flight will be higher and the descent time will be longer; when taking $Q(t)=1 /\left(t_{f}-t+0.5\right)$, the altitude of UAV in the latter period of flight will not be so high, and the variation of UAV flight is smooth. Therefore, considering the simulation results comprehensively, $Q(t)=1 /\left(t_{f}-t+0.5\right)$ is selected as the time function.

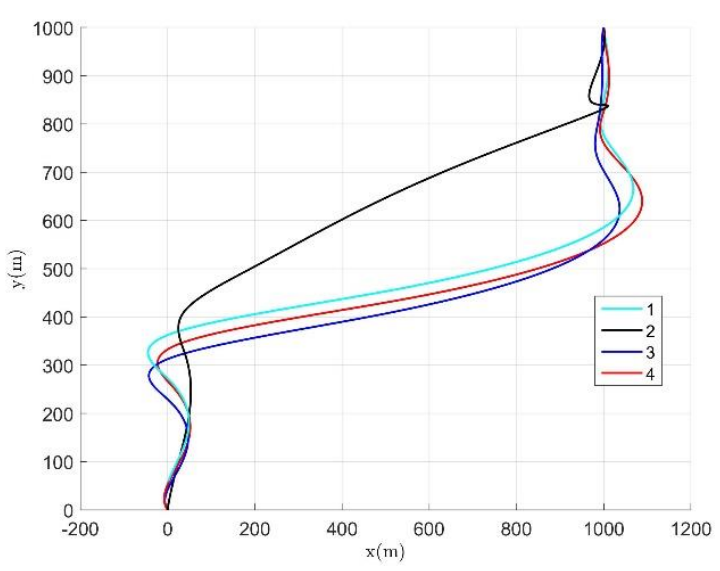

Fig.6 $x-y$ plane flight diagram

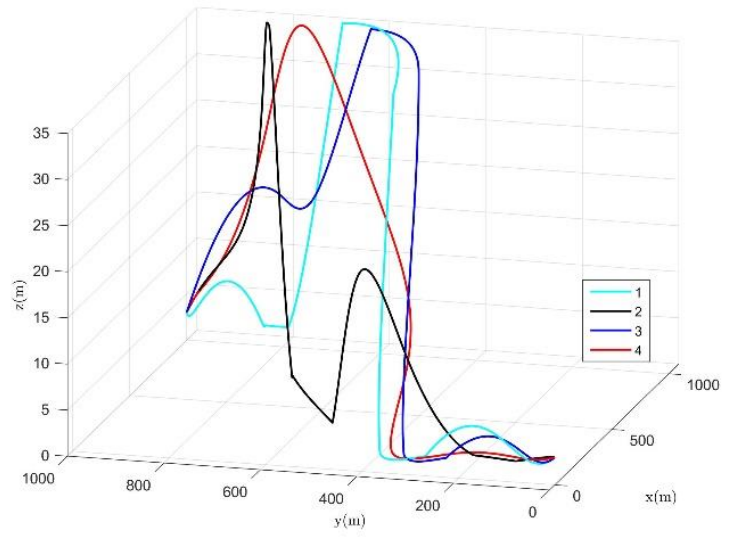

Fig.7 3D flight diagram

\subsection{The problem summary}

Based on the above analysis, we can conclude the following flight path optimization model for formation flight of QUAVs:

For dynamic differential Eq. (1) for QUAV, to find the control quantity to make the performance index Eq. (35) optimal, satisfying boundary value constraints Eq. (15) and Eq. (16), process constraints Eqs. (18-21), (23), (24), (29). 

Now, the problem we faced are:

(1) The optimal solution is a curved form and an infinite dimensional problem.

(2) It is a dynamic equation in differential form.

(3) It is an objective function of integral form.

(4) There are constraints.

To solve these problems, we use Gauss pseudospectral method principle $[33,34]$ to solve:

(1) The optimization problem of the curve is transformed into the optimization problem of several characteristic points on the curve: the infinite-dimensional problem is reduced to a finite dimensional-problem.

(2) The whole curve is fitted by the polynomial.

(3) Transform the differential dynamic equation into algebraic constraint by sampling function.

(4) The integral form of the objective function is transformed into an algebraic equation by using the Gauss quadrature formula.

In this way, the whole optimal control problem is put into the solving framework of nonlinear programming.

\section{Principle of Gauss pseudospectral method}

In Gauss pseudospectral method, control variables and state variables are dispersed first in a selected Gauss point series. Select the discrete points for the node to construct though Lagrange interpolation polynomial, and approximate state and control variables based on the polynomial, and then approximate the derivative of the state variable by taking the derivative of these global interpolation polynomials. The differential equation constraints can be converted into a set of algebraic equation constraints. For the integral term in the performance index, Gauss integral is used for discretization. The terminal state is obtained from the initial state and the integral of the right function, and Gauss integral is used for discretization calculation. After the above transformation, the original optimal control problem is transformed into a parametric optimization problem with a series of algebraic constraints, which is also called Nonlinear Programming problem (NLP) [35-37].

(1) The time-domain transformation

When Gauss pseudospectral method is adopted, the time interval $\left[t_{0}, t_{f}\right]$ needs to be 
transformed into $[-1,1]$, so the time $t$ is converted as follows:

400

$$
t \in\left[t_{0}, t_{f}\right] \rightarrow \tau \in[-1,1], \tau=\frac{2 t}{t_{f}-t_{0}}-\frac{t_{f}+t_{0}}{t_{f}-t_{0}}
$$

401

402

403

404

405

406

407 where,

408

409

$$
\dot{x}(\tau) \approx \dot{X}(\tau)=\sum_{i=0}^{K} D_{k i}\left(\tau_{k}\right) X\left(\tau_{i}\right)
$$

419 where, the differential matrix $D \in R^{K \times(K+1)}$ can be determined offline, namely,

let the approximate state value at the node in Eq. (37) equal to the actual state value, and get $X_{i}=X\left(\tau_{i}\right)=x\left(\tau_{i}\right)$

For the control variables, the same method is used to discretize, that is

$$
u\left(\tau_{k}\right)=U\left(\tau_{k}\right),(k=1, \cdots, K),
$$

namely,

$$
u(\tau) \approx U(\tau)=\sum_{i=1}^{K} L_{i}(\tau) U\left(\tau_{i}\right)
$$

(3) Transformation of dynamic differential equation constraints

Take the derivative of Eq. (37) 


$$
D_{k i}=\dot{L}_{i}\left(\tau_{k}\right)= \begin{cases}\frac{\left(1+\tau_{k}\right) \dot{P}_{K}\left(\tau_{k}\right)+P_{K}\left(\tau_{k}\right)}{\left(\tau_{k}-\tau_{i}\right)\left[\left(1+\tau_{i}\right) \dot{P}_{K}\left(\tau_{i}\right)+P_{K}\left(\tau_{i}\right)\right]} & i \neq k, \\ \frac{\left(1+\tau_{i}\right) \ddot{P}_{K}\left(\tau_{i}\right)+2 \dot{P}_{K}\left(\tau_{i}\right)}{2\left[\left(1+\tau_{i}\right) \dot{P}_{K}\left(\tau_{i}\right)+P_{K}\left(\tau_{i}\right)\right]} & i=k,\end{cases}
$$

421 where, $\tau_{k}(k=1, \cdots, K)$ is a point in the set $\kappa=\left\{\tau_{1}, \tau_{2}, \cdots, \tau_{K}\right\}$, and $\bar{\tau}_{i}(i=0, \cdots, K)$ belongs to 422 the set $\kappa_{0}=\left\{\bar{\tau}_{0}, \bar{\tau}_{1}, \cdots, \bar{\tau}_{K}\right\}$. By substituting Eq. (43) into the dynamic equation, the algebraic 423 constraint equation that the state variable should satisfy on the discrete node can be obtained:

$$
\sum_{i=0}^{K} D_{k i} X\left(\tau_{i}\right)-\frac{t_{f}-t_{0}}{2} f\left(X\left(\tau_{k}\right), U\left(\tau_{k}\right), \tau_{k} ; t_{0}, t_{f}\right)=0
$$

425 where, $k=1, \cdots, K$.

426

(4) Processing of terminal state constraints

The terminal moment node is not included in the approximate expression of the state variable, but the state at the terminal moment should also satisfy the constraint of the dynamic equation:

$$
x\left(\tau_{f}\right)=x\left(\tau_{0}\right)+\int_{-1}^{1} f(x(\tau), u(\tau), \tau) d \tau .
$$

Eq. (45) is approximated by Gauss integral formula. We get

$$
X\left(\tau_{f}\right)=X\left(\tau_{0}\right)+\frac{t_{f}-t_{0}}{2} \sum \omega_{k} f\left(X\left(\tau_{k}\right), U\left(\tau_{k}\right), \tau ; t_{0}, t_{f}\right),
$$

where, $\omega_{k}=\int_{-1}^{1} L_{i}(\bar{\tau}) d \tau$ is Gauss weight, $\tau$ is Legendre-Gauss point.

(5) Approximation of integral term in the performance index function

The integral term in the performance index function is approximated by the Gauss integral formula:

$$
J=\Phi\left(X_{0}, t_{0}, X_{f}, t_{f}\right)+\frac{t_{f}-t_{0}}{2} \sum_{k=1}^{2} w_{k} g\left(X_{k}, U_{k}, \tau_{k} ; t_{0}, t_{f}\right)
$$

where, $\Phi()$ is the no-integral term in the performance index function.

(6) General description of the Gauss pseudospectral method

Based on the above mathematical transformation, the principle of Gauss pseudospectral method to solve the optimal control problem can be described as follows: calculate state variables $X_{i}, i=0, \cdots, K$ and control variables $U_{k}, k=1, \cdots, K$ and $t_{0}, t_{f}$ on the discrete nodes, to make the minimum performance index Eq. (47), and satisfy the dynamics equation of algebraic constraint 
443

Eq. (44), the terminal state constraint Eq. (46), and the boundary conditions of the original optimal control problem

$$
\varphi\left(X_{0}, t_{0}, X_{f}, t_{f}\right)=0
$$

and process constraints

$$
C\left(X_{k}, U_{k}, \tau_{k} ; t_{0}, t_{f}\right) \leq 0, \quad k=1, \cdots, K
$$

thus, the original optimal control problem is transformed into a nonlinear programming problem (NLP), namely:

$$
\begin{array}{ll}
\min & F(y), y \in R^{M}, \\
\text { s.t. } & g_{j}(y) \geq 0, \quad j=1,2, \cdots, p, \\
& h_{j}(y)=0, \quad j=1,2, \cdots, l .
\end{array}
$$

where, $y$ is a designed variable, including control variable, state variable and two endpoint time.

At present, there are many effective methods to solve the nonlinear programming problem, among which the sequential quadratic programming algorithm (SQP) is widely used for its local superone-time convergence and global convergence, so the Gauss pseudospectral method uses SQP algorithm to deal with the transformed NLP problem.

\section{The simulation verification}

The simulation in this paper is aimed at the formation of six QUAVs. The type of UAVs we used is the ZY F150 QUAV. When taking off, the formation is formed by equilateral triangle, the formation keeping strategy is the leader-followed method. The relevant parameters of the QUAVs used in the experiment are shown in Eq. (51) and Tab.1.

$$
\left\{\begin{array}{l}
0 \leq x_{i} \leq 1500 \mathrm{~m}, 0 \leq y_{i} \leq 1500 \mathrm{~m}, 0 \leq z_{i} \leq 35 \mathrm{~m}, \\
\left|v_{x i}\right| \leq 5 \mathrm{~m} / \mathrm{s},\left|v_{y i}\right| \leq 5 \mathrm{~m} / \mathrm{s},\left|v_{z i}\right| \leq 2 \mathrm{~m} / \mathrm{s}, \\
\left|\phi_{i}\right| \leq \pi,\left|\theta_{i}\right| \leq \pi / 3,\left|\psi_{i}\right| \leq \pi / 3, \\
\left|p_{i}\right| \leq 32^{\circ} / \mathrm{s},\left|q_{i}\right| \leq 10^{\circ} / \mathrm{s},\left|r_{i}\right| \leq 5^{\circ} / \mathrm{s}, \\
0 \leq U_{1 i} \leq 5.2 \mathrm{~N}, \\
\left|U_{2 i}\right| \leq 0.0501 \mathrm{~N} \cdot \mathrm{m}, \\
\left|U_{3 i}\right| \leq 0.0501 \mathrm{~N} \cdot \mathrm{m}, \\
\left|U_{4 i}\right| \leq 0.0209 \mathrm{~N} \cdot \mathrm{m} .
\end{array}\right.
$$


Table 1 Simulation parameters of the ZY F150 QUAV

\begin{tabular}{|c|c|c|c|}
\hline Symbol & Physical meaning & Value & Unit \\
\hline$m$ & Mass of the quadrotor & 0.5 & $\mathrm{~kg}$ \\
\hline$l$ & $\begin{array}{l}\text { Length between the center of the aircraft } \\
\text { and the rotor }\end{array}$ & 0.075 & $\mathrm{~m}$ \\
\hline$K_{T}$ & Lift coefficient & $1.105 \mathrm{e}-5$ & $\mathrm{~N} /(\mathrm{rad} / \mathrm{s})^{2}$ \\
\hline$K_{M}$ & Inverse torque coefficient & $1.779 \mathrm{e}-7$ & $\mathrm{~N} \cdot \mathrm{m} /(\mathrm{rad} / \mathrm{s})^{2}$ \\
\hline$I_{x x}$ & Rotational inertia of the $\mathrm{x}$-axis & 0.0219 & $\mathrm{~kg} \cdot \mathrm{m}^{2}$ \\
\hline$I_{y y}$ & Rotational inertia of the $y$-axis & 0.0219 & $\mathrm{~kg} \cdot \mathrm{m}^{2}$ \\
\hline$I_{z z}$ & Rotational inertia of the z-axis & 0.0366 & $\mathrm{~kg} \cdot \mathrm{m}^{2}$ \\
\hline$g$ & Gravitational acceleration & 9.8 & $\mathrm{~m} / \mathrm{s}^{2}$ \\
\hline$K_{1}, K_{2}, K_{3}$ & Coefficient of air resistance & 0.073 & $\mathrm{~N} /(\mathrm{m} / \mathrm{s})^{2}$ \\
\hline$K_{4}, K_{5}, K_{6}$ & Coefficient of air resistance moment & 0.0055 & $\mathrm{~N} \cdot \mathrm{m} /(\mathrm{rad} / \mathrm{s})^{2}$ \\
\hline
\end{tabular}

The terrain constraint model adopts the ground height generated by Eq. (52) and the mountain terrain data generated by Eq. $(53)[38,39]$.

$$
\begin{gathered}
z_{1}(x, y)=\operatorname{abs}\left(\sin (y+a)+b \cdot \sin (x)+c \cdot \cos \left(d \cdot \sqrt{x^{2}+y^{2}}\right)+e \cdot \cos (y)+f \cdot \sin \left(g \cdot \sqrt{x^{2}+y^{2}}\right)\right) \\
z_{2}(x, y)=\sum_{i=1}^{n} h_{i} \exp \left[-\left(\frac{x-x_{i}}{x_{s i}}\right)^{2}-\left(\frac{y-y_{i}}{y_{s i}}\right)^{2}\right]
\end{gathered}
$$

where, in Eq. (52), $x$ and $y$ are the point coordinates of the model projected on the horizontal plane, $z_{1}$ is the ground surface elevation value corresponding to the horizontal surface point, $a, b, c, d$, $e, f, g$ are the constant coefficients to control the rise and fall of the ground surface reference terrain in the digital map model, function abs() represents absolute function, that is, all terrain heights are above sea level, as the reference terrain of the formation environment of the QUAVs. This paper takes $x=y=\left[\begin{array}{lll}0 & 0.001 & 0.002 \ldots 1500\end{array}\right], a=10, b=0.2, c=0.1, d=0.6, e=1, f=0.1, g=0.1$. In Eq. (53), $z_{2}$ is the elevation value given at the corresponding point $(x, y)$ on the map, $\left(x_{i}, y_{i}\right)$ is the central coordinate of the $i$-th mountain, $h_{i}$ is the terrain parameter to controls the height of the 
mountain, $x_{s i}$ and $y_{s i}$ are the attenuation of the $i$-th mountain along the $x$-axis and $y$-axis respectively, which controls the slope, and $n$ represents the total number of mountains. By setting the above parameters, different number of peaks with different heights and slopes can be achieved. This

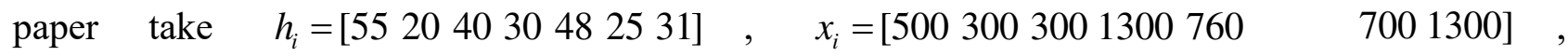
$y_{i}=\left[\begin{array}{lllll}11004108004507502001300\end{array}\right] \quad, \quad x_{s}=\left[\begin{array}{lllll}50 & 403086876030\end{array}\right] \quad, \quad y_{s}=\left[\begin{array}{lll}50 & 30\end{array}\right.$ 20798390 30], so there are seven peaks.

In the formation of UAVs with leader-followed strategy, the reference path of the leader UAV should be specified first, and the method proposed in this paper is used to generate the reference path of the leader UAV. The path generated by GPM is given in the form of nodes. In this paper, B-spline smoothing strategy is adopted to smooth the process and limit the maximum value.

Simulation 1: Path planning of the formation of UAV when $0 \leq z_{i} \leq 35 \mathrm{~m}$

When we limit the maximum height that a quadcopter can fly to as its maximum height $z_{i}=35 \mathrm{~m}$, the task to be accomplished by the leader UAV is to start from the specified starting point $\left(x_{0}, y_{0}, z_{0}\right)=(2,1,3) \mathrm{m}$, with the initial velocity vector $\left(v_{x 0}, v_{y 0}, v_{z 0}\right)=(2,2,0) \mathrm{m} / \mathrm{s}$ and the initial attitude angle $\left(\psi_{0}, \theta_{0}, \phi_{0}\right)=\left(45^{\circ},-1^{\circ}, 0^{\circ}\right)$. It passes through the non-flat area distributed over a number of mountains, and arrive at the specified location $(1200,1000,2) \mathrm{m}$ with the specified velocity vector $\left(v_{x f}, v_{y f}, v_{z f}\right)=(0,0,0) \mathrm{m} / \mathrm{s}$ and attitude angle $\left(\psi_{f}, \theta_{f}, \phi_{f}\right)=\left(45^{\circ}, 0^{\circ}, 0^{\circ}\right)$ at the specified time $t=275 \mathrm{~s}$, and gradually descend from the high altitude to the specified height $2 \mathrm{~m}$. The take-off positions of the five followed UAVs are $(0,0,3) \mathrm{m},(0,2,3) \mathrm{m},(1,0.5,3) \mathrm{m}$, $(0,0.5,3) \mathrm{m},(1,1.5,3) \mathrm{m}$, respectively. The take-off velocity vector, attitude angle, terminal velocity vector and attitude angle are the same as the leader UAVs. The simulation result is shown in Fig. $8 \sim$ Fig. 14 .

Fig. 8 and Fig. 9 show the flight path from different visual angles of the formation of UAVs in the environment with multiple obstacles distributed, and it can be seen that it does not collide with any obstacles and can reach the specified end point from the starting point. As can be seen from Fig. 
8, the flight path is smooth throughout the flight, and all of which meet the constraints; due to large maximum flying height of the ZY F150 QUAV, the take-off point nearest obstructions can be directly overflew, and then keep flying at a certain height, don't need to turn to evade, which can prevent again to evade other obstacles; in the followed flight process, UAVs formation by changing the flight direction and height to consume the excess flight time. Also, we can see that, after crossing the first obstacle, the flight path of UAV formation is far from other obstacles, so UAVs don't need to transform formation. However, from the perspective of energy consumption, the landing process can save energy consumption while consuming extra time compared with the horizontal circling flight. At the end of flight, it can be seen that the formation of UAV gradually lands at the specified altitude of $2 \mathrm{~m}$ from the particular direction. Fig. 9 also shows the change of the heading angle of the leader UAV. The pitch angle and roll angle are showed in Fig. 10.

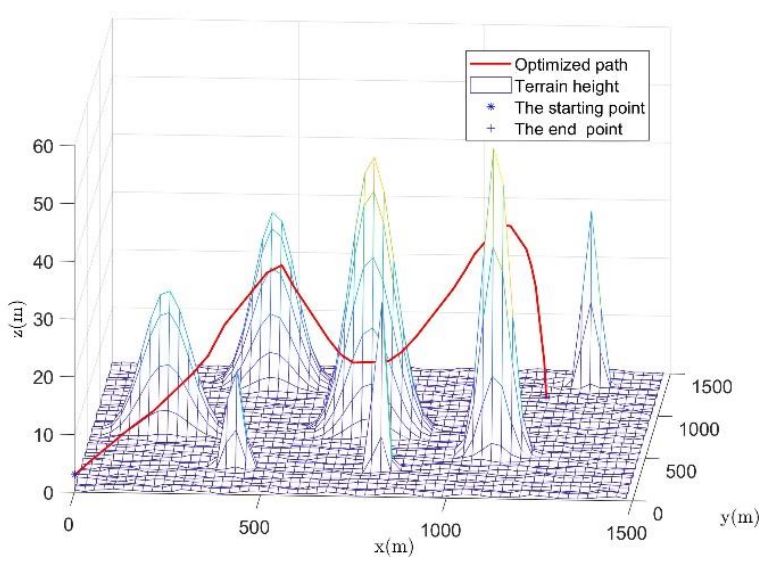

Fig. $83 \mathrm{D}$ view of the optimized path

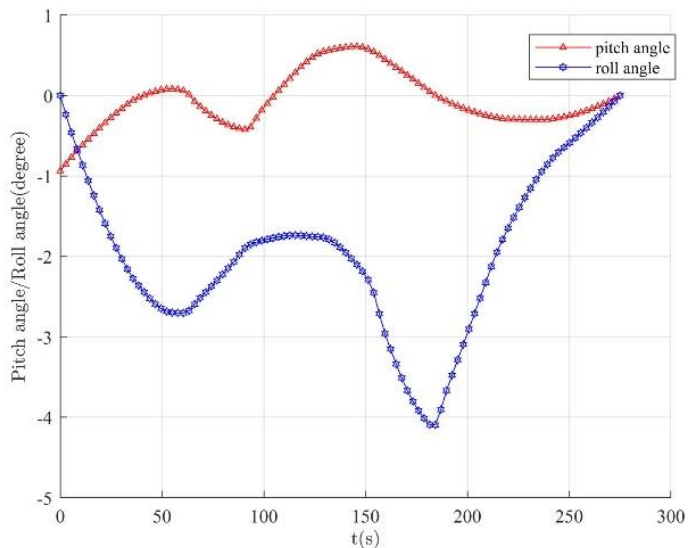

Fig. 10 The curves of pitch angle and roll angle of the leader UAV

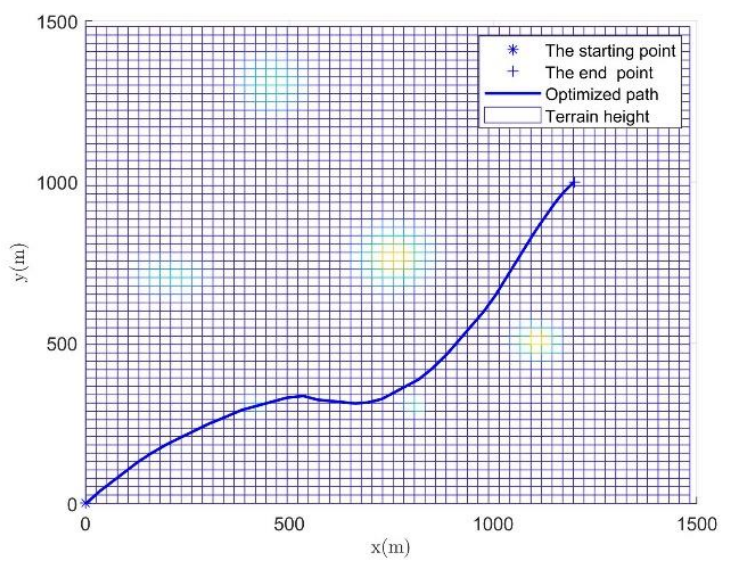

Fig. $9 x-y$ plane of the optimized path

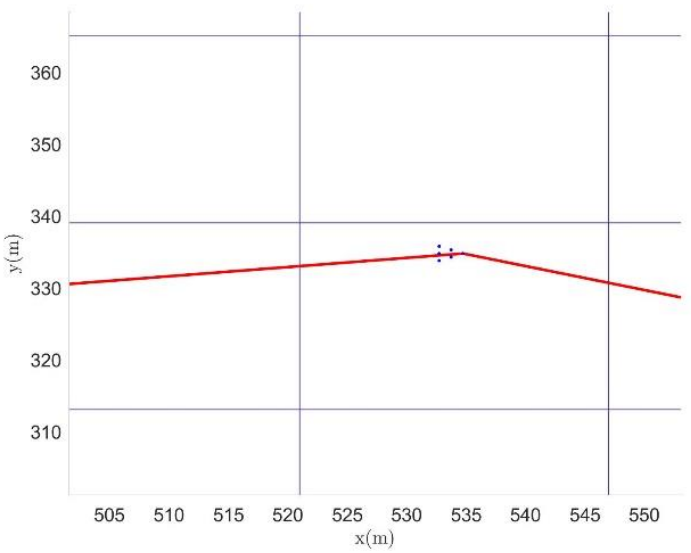

Fig. 11 Formation of UAVs

From Fig. 10 we can see that the change of pitch angle and roll angle of the leader UAV is small 
during the entire flight, this is also related to the characteristics of the QUAV, that to change the heading angle, ascent or descent of a QUAV does not need to change its pitch angle, and because the whole flight process is in a higher airspace, there is no need to roll to avoid obstacles or change formation, so the roll angle of the whole flight process is also small. The enlarged figure is shown in Fig.11, which shows the formation of QUAVs during the flying process (The dots represent the six QUAVs).

Fig. 12 and Fig. 13 show the curves of variation of control variables $U_{1}, U_{2}, U_{3}$ and $U_{4}$, respectively. It can be seen from Fig. 12 that $U_{1}$ is used to change the speed of the three spatial directions to realize the change of the trajectory of the leader UAV, so as to avoid obstacles or consume extra time for the leader UAV. It can be seen from Fig.13 that roll angle control quantity keeps a small value in the entire process of flight, which shows that the roll angle of the leader UAV almost does not changed during the flight; in the process of flight, the pitch angle control quantity is also small, although there are up and down, but due to the special structure of QUAV, the pitch angle does not needed to be great changed, which also accord with the results of simulation; but the change of yaw angle control quantity is bigger, because it need to change the flight direction of leader UAV.

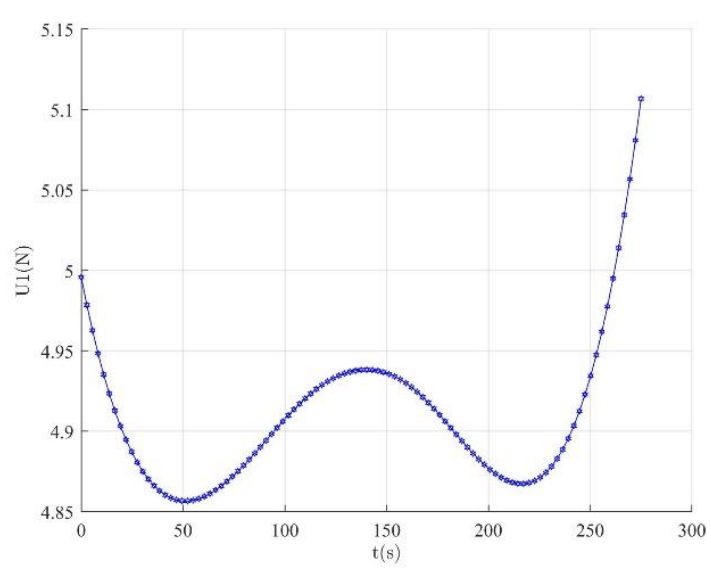

Fig. 12 The curves of $U_{1}$ of the leader UAV

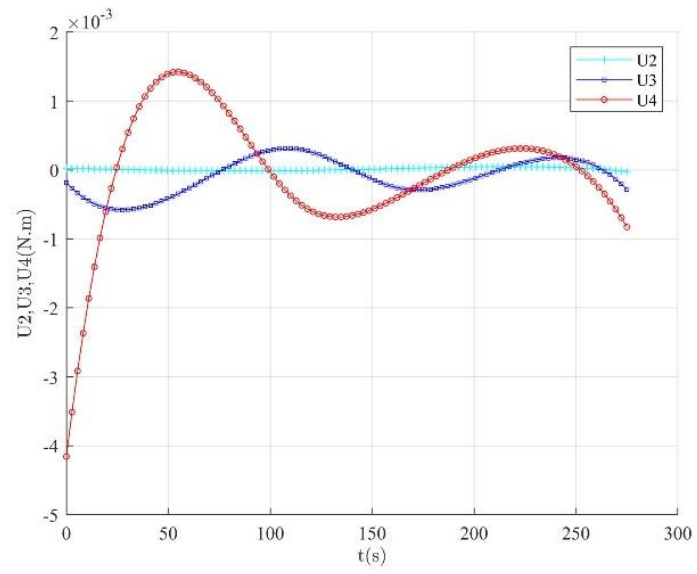

Fig. 13 The curves of $U_{2}, U_{3}$ and $U_{4}$ of the leader UAV

Fig. 14 shows the curves of variation of linear velocity variables $v_{x}, v_{y}$ and $v_{z}$ respectively. Since we are using the NED coordinate system, the change of the $Z$ axis direction is the opposite of the normal situation. It can be seen from Fig. 14 that when the vertical velocity $v_{z}$ changes, the horizontal velocity can be changed through the change of $v_{x}$ and $v_{y}$, so as to realize the UAV's obstacle avoidance or consume extra flight time in the corresponding $x-y$ plane. 


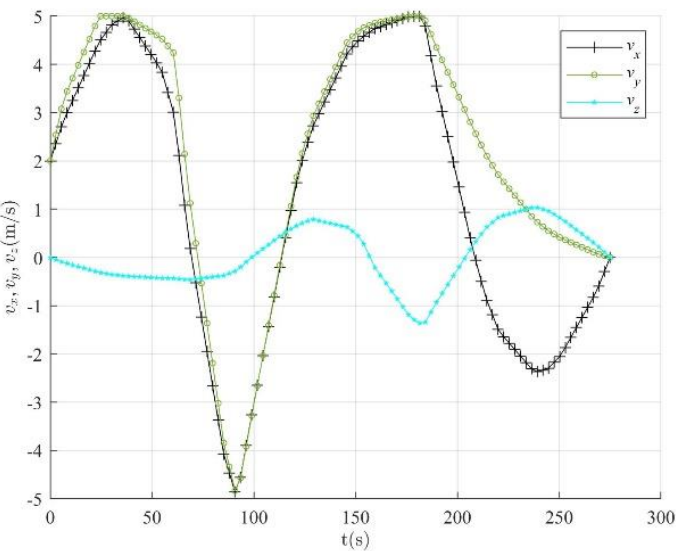

Fig. 14 The curves of $v_{x}, v_{y}$ and $v_{z}$ of the leader UAV

Simulation 2: Path planning of the formation of UAV when $0 \leq z_{i} \leq 15 \mathrm{~m}$

When we limit the maximum height that the QUAV can fly to as $z_{i}=15 \mathrm{~m}$, the task to be accomplished by the leader UAV is to start from the specified starting point $\left(x_{0}, y_{0}, z_{0}\right)=(2,1,3) \mathrm{m}$, with the initial velocity vector $\left(v_{x 0}, v_{y 0}, v_{z 0}\right)=(2,2,0) \mathrm{m} / \mathrm{s}$ and the initial attitude angle $\left(\psi_{0}, \theta_{0}, \phi_{0}\right)=\left(60^{\circ},-18^{\circ}, 0^{\circ}\right)$, and arrive at the specified location $(1200,1000,2) \mathrm{m}$ with the specified velocity vector $\left(v_{x f}, v_{y f}, v_{z f}\right)=(0,0,0) \mathrm{m} / \mathrm{s}$ and attitude angle $\left(\psi_{f}, \theta_{f}, \phi_{f}\right)=$ $\left(-60^{\circ}, 0^{\circ}, 0^{\circ}\right)$ at the specified time $t=350 \mathrm{~s}$. The simulation result is shown in Fig. $15 \sim$ Fig. 20.

550

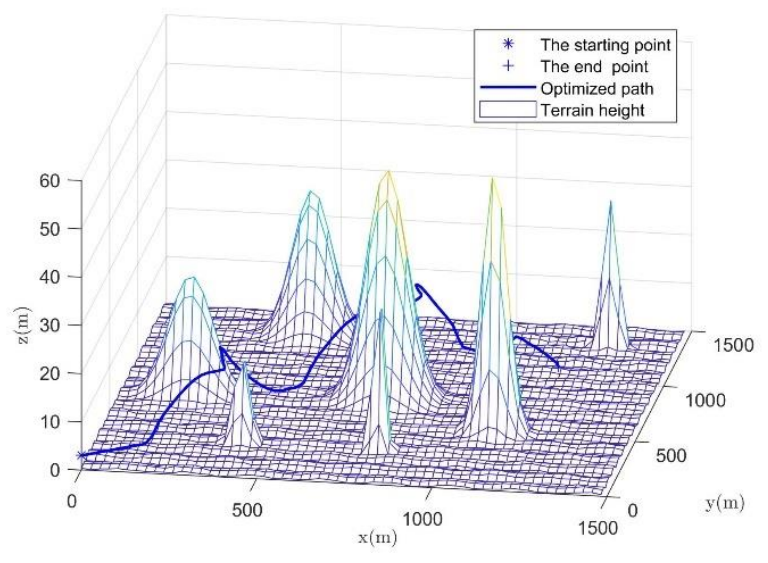

Fig. $153 \mathrm{D}$ view of the optimized path

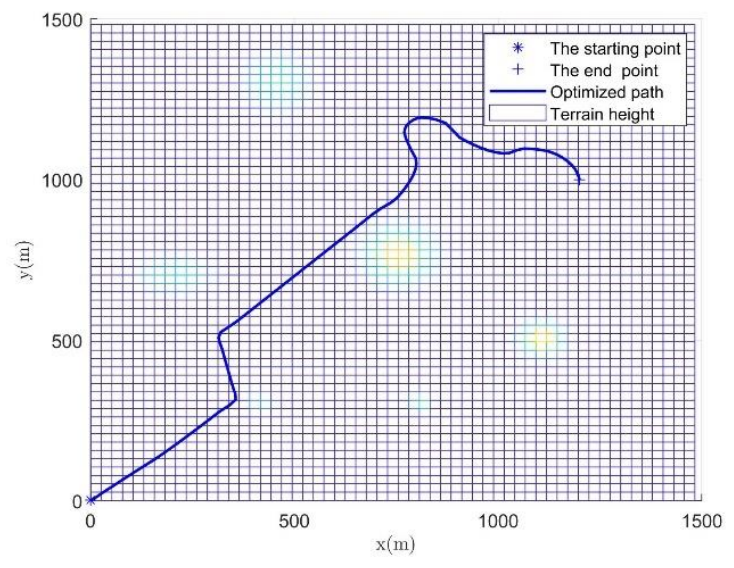

Fig. $16 x-y$ plane of the optimized path

As can be seen from Fig. 15, when the maximum height that the QUAV can fly to is limited as $z_{i}=15 \mathrm{~m}$, it can't fly over the mountain and has to avoid the need to detour. From the optimization path in $x-y$ plane graph in Fig. 16, we can see that the UAV formation need to change flight course 
between peaks, and ultimately to heading to the end point, which is significant different from Simulation-1.However, as we stipulate that the UAV formation should reach the target point at the specified time of $350 \mathrm{~s}$, so the UAV formation needs to make some detours in the flight process to consume the extra flight time, which is also automatically considered in the optimization process. Of course, we can also consider the time optimization as the optimization goal. Fig. 16 also shows the change of the heading angle of the leader UAV. The pitch angle and roll angle are showed in Fig. 17.

From Fig. 17 we can see that the change of pitch angle of the leader UAV is also small during the whole flight, but because there are some turns to avoid obstacles or to change formations during the whole flight process, so the roll angle of the whole flight process is a little larger than the value in Simulation 1.

Fig. 18 shows the curves of variation of linear velocity variables $v_{x}, v_{y}$ and $v_{z}$, respectively. It can be seen from Fig. 18 that as we reduce the maximum flying height of the UAV, the change of $v_{z}$ in the Z-axis direction is also smaller than that in Simulation 1. However, due to many changes in the flight direction, the change of $v_{x}$ and $v_{y}$ in the horizontal direction is more, and the change of $v_{x}$ and $v_{y}$ is not consistent as that in Simulation 1.

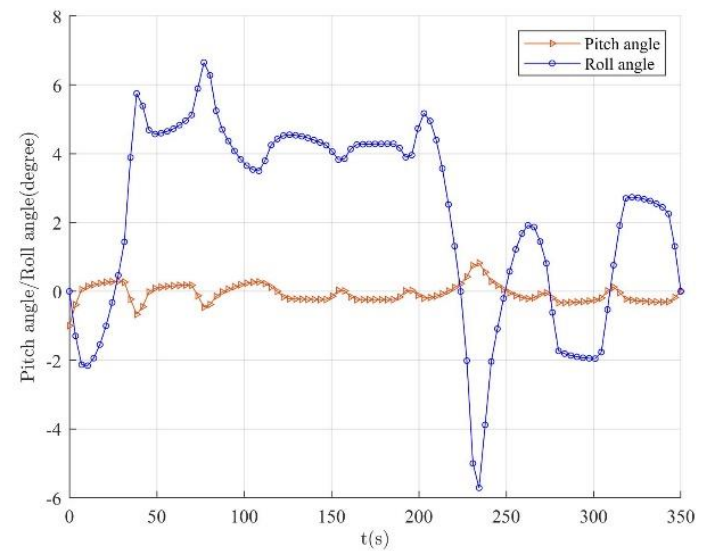

Fig. 17 The curves of pitch angle and roll angle Fig. 18 The curves of $v_{x}, v_{y}$ and $v_{z}$ of the leader UAV of the leader UAV

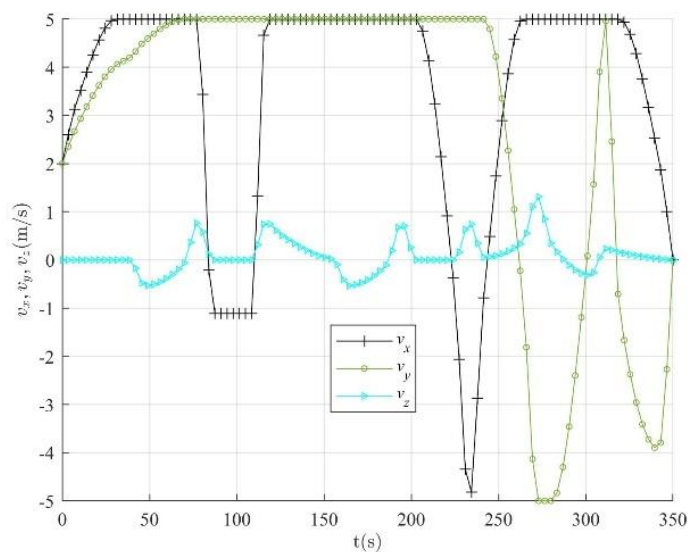
respectively. It can be seen from Fig. 19 and Fig. 20, relatively speaking, due to the long flight time of UAV formation, the value of $U_{1}$ is smaller than $U_{1}$ in Simulation-1, but the control quantity $U_{2}$, 

Simulation-1.

579

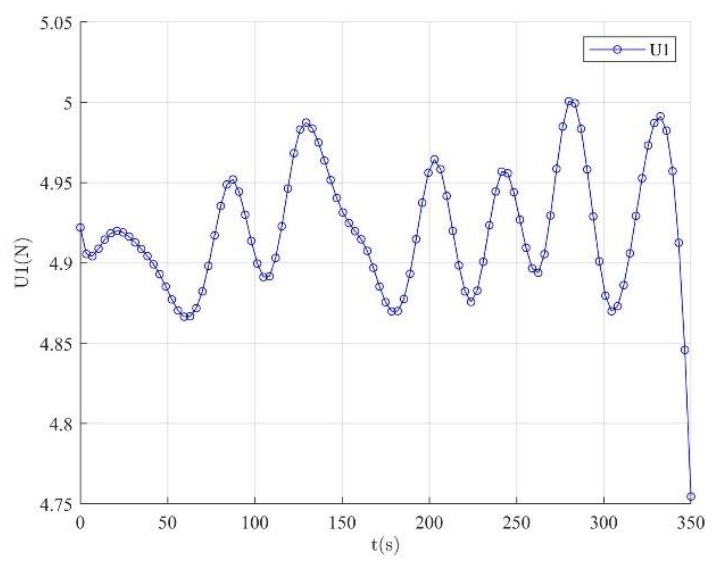

Fig. 19 The curves of $U_{1}$ of the leader UAV

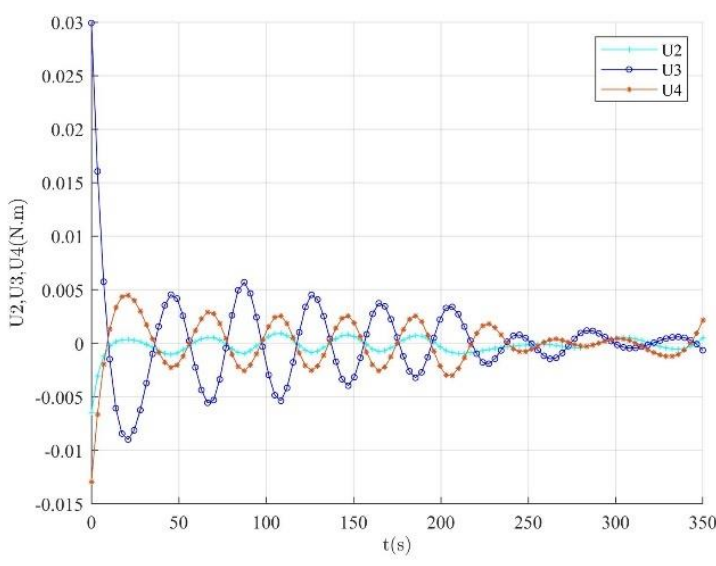

Fig. 20 The curves of $U_{2}, U_{3}$ and $U_{4}$ of the leader UAV

\section{5 conclusion}

In this paper, the formation problem of multi-QUAVs was studied. Firstly, the strategy of the leader-followed method was adopted, and the formation keeping problem between each QUAV was defined based on the consistency theory. The formation system was transformed into a network topology, and then the formation was treated as a time-varying virtual rigid body by using the virtual structure method. When a UAV formation encounters an obstacle, it sometimes needs to change its formation to get through the obstacle. Formation transformation in the flight of UAVs formation can be regarded as reassembly of UAVs, namely formation reconstruction. The methods of formation rotation, formation reduction or new formation were introduced in this paper. On this basis, firstly, some constraints of the leader QUAV were analyzed. Then, by analyzing the constraint information of the followed UAVs in the formation, such as the position of the followed UAVs in the formation and the minimum turning radius of the followed UAVs, the information of the followed UAVs in the formation was converted to constraints of the leader UAV, so that the formation problem can be isolated. After that, the requirement of performance index was analyzed, if a UAV formation is required to fly in a straight line from a certain horizontal direction at a certain altitude to the target point, so the terminal flight requirement was added, and the traditional performance index was improved. Then this paper gave the analysis of the formation path optimization of the QUAV, summarized the final problems of path optimization, namely infinite dimension, differential, integral, 
599

600

601

602

603

604

605

606

607

608

609

610

611

612

613

614

615

616

617

618

619

620

621

622

623

624

constrained, etc, and proposed the Gauss pseudospectral method to transform the optimal control problem of the path optimization into the solving framework of nonlinear programming. Finally, we conducted two simulations for different maximum flight height. The simulation results showed the effectiveness of the proposed method in this paper.

\section{Acknowledgments}

This work is supported by the National Natural Science Foundation of China (No. 61873186), and the Key R\&D and Promotion Projects (tackling of key scientific and technical problems) in Henan Province, China (No. 212102210497).

\section{Conflicts of interest/Competing interests}

The authors declare that they have no conflict of interest.

\section{References:}

[1] Hafez A. T., Kamel M. A. Cooperative task assignment and trajectory planning of unmanned systems via HFLC and PSO. Unmanned Systems, 2019, 7(2):65-81.

[2] Qi G., Huang D., Modeling and dynamical analysis of a small-scale unmanned helicopter. Nonlinear Dynamics, 2019, 98(3): 2131-2145.

[3] Manyam G. S., Rathinam S., Casbeer D., et al. Tightly bounding the shortest Dubins paths through a sequence of points. Journal of Intelligent and Robotic Systems, 2017, 88: 495-511.

[4] Ropero F., Munoz P., R-Moreno M. D. A path planning algorithm for cooperative UGV-UAV exploration. Engineering Applications of Artificial Intelligence, 2019, 78(FEB.): 260-272.

[5] Liu C., Mcaree O. and Chen W. H. Path-following control for small fixed - wing unmanned aerial vehicles under wind disturbances. International Journal of Robust \& Nonlinear Control, 2013, 23(15):1682-1698.

[6] Radmanesh M., Kumar M., Guentert P. H., et al. Overview of path planning and obstacle avoidance algorithms for UAVs: a comparative study. Unmanned Systems, 2018 6, (2):1-24.

[7] Chen Y., Yu J., Su X., et al. Path planning for multi-UAV formation. Journal of Intelligent and Robotic Systems, 2015, 77: 229-246. 
[8] Ergezer H., Leblebicioglu K. 3D Path planning for multiple UAVs for maximum information collection. Journal of Intelligent and Robotic Systems, 2014, 73:737-762.

[9] Zhou S., Qi Y., Zhang L., et al. Time-varying formation control of UAV swarm systems with switching topologies. Acta Aeronautica et Astronautica Sinica, 2017, 38(4):259-267(in Chinese).

[10] Nigam N., Bieniawski S., Kroo I., et al. Control of multiple UAVs for persistent surveillance: algorithm and flight test results. IEEE Transactions on Control Systems Technology, 2012, 20(5):1236-1251.

[11] Turpin M., Michael N., Kumar V. Trajectory design and control for aggressive formation flight with quadrotors. Autonomous Robots, 2012, 33(1-2):143-156.

[12] Turpin M., Michael N., Kumar V. Decentralized formation control with variable shapes for aerial robots// Proceeding Conference of 2012 IEEE International Conference on Robotics and Automation, Saint Paul, MN, 2012: 23-30.

[13] Shanmugavel M., Tsourdos A., White B., et al. Co-operative path planning of multiple UAVs using Dubins paths with clothoid arcs. Control Engineering Practice, 2010, 18(9):1084-1092.

[14] Brintaki A. N., Nikolos I. K. Coordinated UAV path planning using Differential Evolution. Operational Research, 2005, 5(3):487-502.

[15] Besada-Portas E., Dela T. L., De la C. J. M., et al. Evolutionary trajectory planner for multiple UAVs in realistic scenarios. IEEE Transactions on Robotics, 2010, 26(4):619-634.

[16] Radmanesh R., Kumar M., French D., et al. Towards a PDE-based large-scale decentralized solution for path planning of UAVs in shared airspace. Aerospace ence and Technology, 2020:105965.

[17] Chen Y., Yu J., Su X., et al. Path planning for multi-UAV formation. Journal of Intelligent \& Robotic Systems, 2015, 77(1):229-246.

[18] Zhang L., Sun M., Cheng Q., et al. Integral Performance Optimization for the Two-Stage-toOrbit RBCC-RKT Launch Vehicle Based on GPM [J], The Aeronautical Journal, 2019, 123(1265): 945-969.

[19] Yang S., Cui T., Hao X., et al. Trajectory optimization for a ramjet-powered vehicle in ascent phase via the Gauss pseudospectral method. Aerospace Science and Technology, 2017, 67: 8895.

[20] Zhuang J., Zhang Q., Zhang J., et al. Flat Trajectory Optimization of Tilt-rotor Aircraft Based on 
655

656

657

658

659

660

661

662

663

664

665

666

667

668

669

670

671

672

673

674

675

676

677

678

679

680

681

682

683

684

Gauss Pseudospectral Method. Journal of Physics: Conference Series. IOP Publishing, 2020, 1624(4): 042033.

[21] Bellahcene Z., Bouhamida M., Denai M., et al. Adaptive neural network-based robust $\mathrm{H} \infty$ tracking control of a quadrotor UAV under wind disturbances[J]. International Journal of Automation and Control, 2021, 15(1):28.

[22] Bi H., Qi G., Hu J., et al. Hidden and transient chaotic attractors in the attitude system of quadrotor unmanned aerial vehicle. Chaos Solitons \& Fractals, 2020, 138: 109815.

[23] Bi H., Qi G. and Hu J. Modeling and analysis of chaos and bifurcations for the attitude system of a quadrotor unmanned aerial vehicle. Complexity, 2019, 2019: 1-17.

[24] Farid G., Mo H., Ahmed M. I., et al. On control law partitioning for nonlinear control of a quadrotor UAV. Proceeding Conference of International Bhurban Conference on Applied and Science \& Technology, Islamabad, Pakistan, 2018: 257-262.

[25] Liu Y., Chen C., Wu H., et al. Structural stability analysis and optimization of the quadrotor unmanned aerial vehicles via the concept of Lyapunov exponents. International Journal of Advanced Manufacturing Technology, 2018(94): 3217-3227.

[26] OMüRLü V. E., AHIN U. B., Artar R., et al. An experimental stationary quadrotor with variable DOF. Sadhana. Academy Proceedings in Engineering Sciences, 2013, 38(2): 247-264.

[27] Turpin M., Michael N., Kumar V. Trajectory design and control for aggressive formation flight with quadrotors. Autonomous Robots, 2012, 33(1-2): 143-156.

[28] Du H., Zhu W., Wen G., et al. Distributed formation control of multiple Quadrotor aircraft based on nonsmooth consensus algorithms. IEEE Transactions on Cybernetics, 2017:1-12.

[29] Kolaric P., Chen C., Dalal A., et al. Consensus controller for multi-UAV navigation. Control Theory \& Technology, 2018, 16(2): 110-121.

[30] Dong X., Yu B., Shi Z., et al. Time-varying formation control for unmanned aerial vehicles: theories and applications. IEEE Transactions on Control Systems Technology, 2014, 23(1): 340348.

[31] Wei G., Jun T., Liang B., et al. Time synergistic optimal efficiency model for formation transformation of multiple UAVs. Acta Aeronautica et Astronautica Sinica, 2019, 40(6): 322599.1-9. (in Chinese)

[32] Zhang L., Sun M., Chen Z., et al. Trajectory planning with determinate impact angle and time 
constraints based on pseudospectral method. ournal of Balls, 2013, 25(3): 1-5+12. (in Chinese)

[33] Huntington G. T. Advancement and analysis of a Gauss pseudospectral transcription for optimal control problems. Boston: Massachusetts Institute of Technology, 2007.

[34] Zhang L., Gao H., Chen Z., et al. Multi-objective global optimal parafoil homing trajectory optimization via Gauss pseudospectral method. Nonlinear Dynamics, 2013, 72(3): 1-8.

[35] Huntington G. T., Rao A. V. Optimal reconfiguration of spacecraft formations using the Gauss pseudospectral method. Journal of Guidance Control \& Dynamics, 2012, 31(3):689-698.

[36] Chen Q., Wang X., Yang J. Optimal path-following guidance with generalized weighting functions based on indirect Gauss pseudospectral method. Mathematical Problems in Engineering, 2018, 2018(PT.10):3104397.1-3104397.17.

[37] Xiao L., Lv L., Liu P., et al. A novel adaptive Gauss pseudospectral method for nonlinear optimal control of constrained hypersonic re-entry vehicle problem. International Journal of Adaptive Control and Signal Processing, 2018, 32(9):1243-1258.

[38] Nikolos I. K., Valavanis K. P., Tsourveloudis N. C., et al. Evolutionary algorithm based offline/online path planner for UAV navigation. IEEE Transactions on Systems Man \& Cybernetics Part BCybernetics A Publication of the IEEE Systems Man \& Cybernetics Society, 2003, 33(6):898-912.

[39] Zhang Y., Yang X., Zhou W., et al. Study of three-dimensional on-line path planning for UAV based on pythagorean hodograph curve. International Journal on Smart Sensing and Intelligent Systems, 2015, 8(3):1641-1666. 
Figures

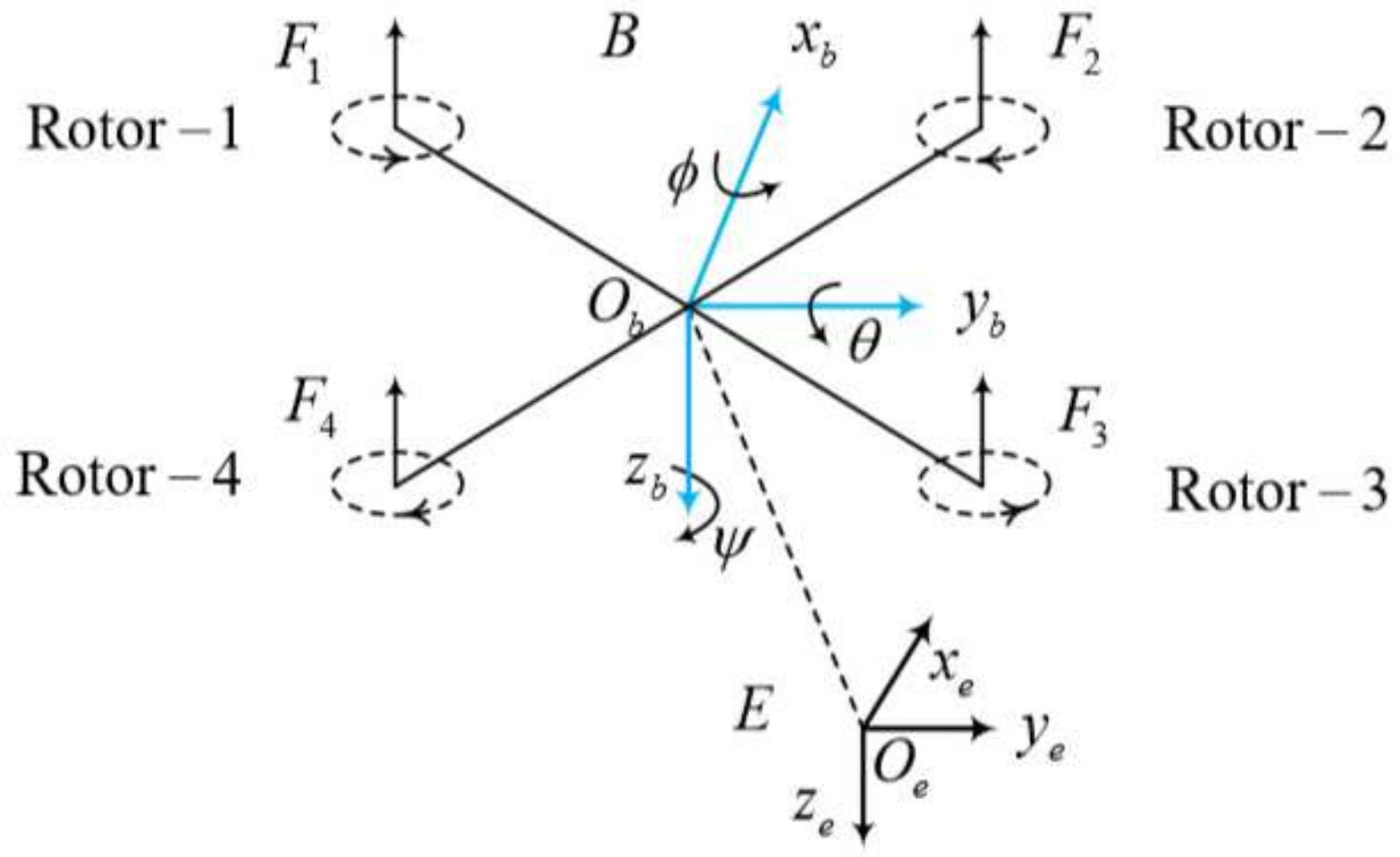

Figure 1

Schematic view of the QUAV system. 


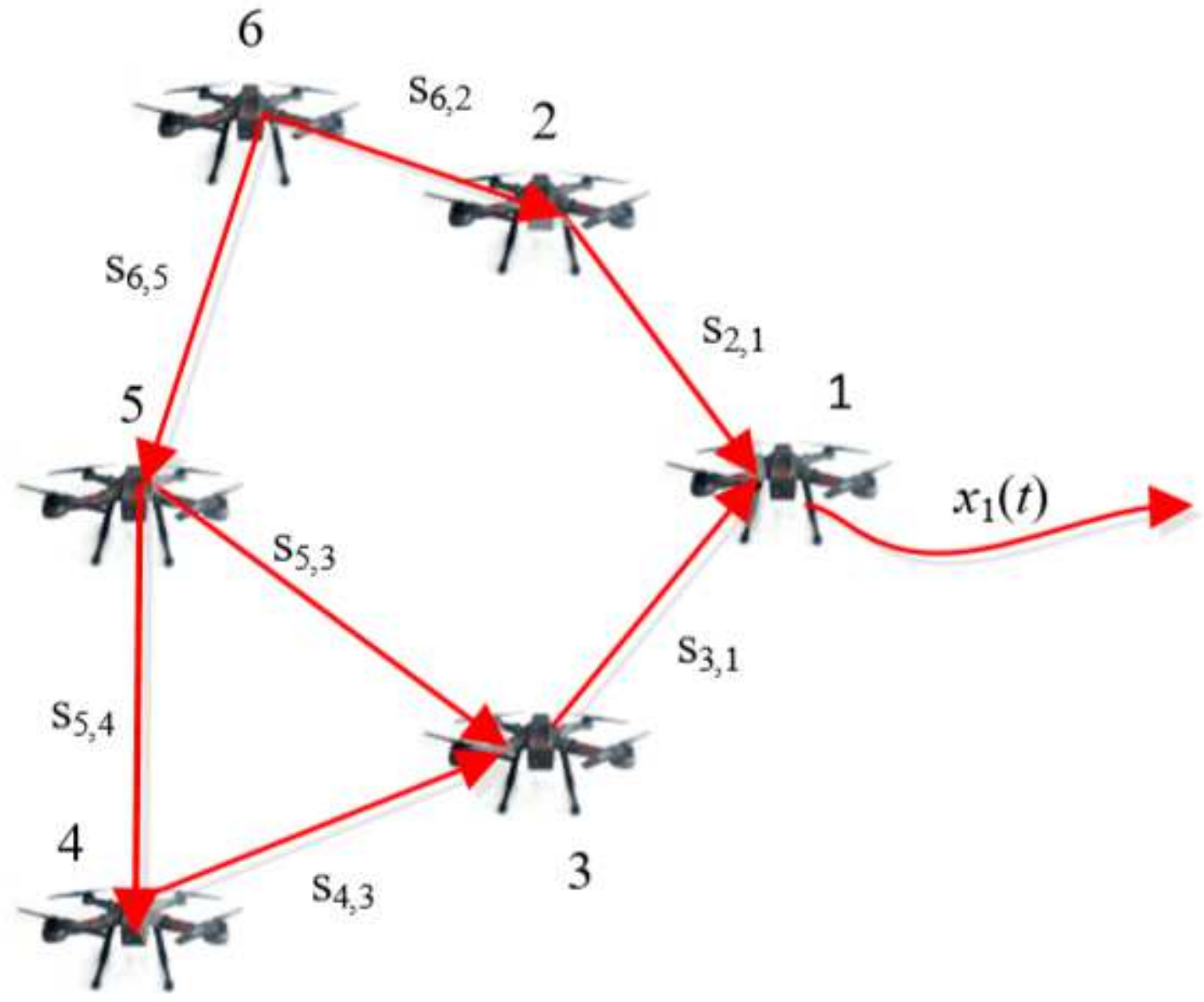

Figure 2

Formation of QUAVs 

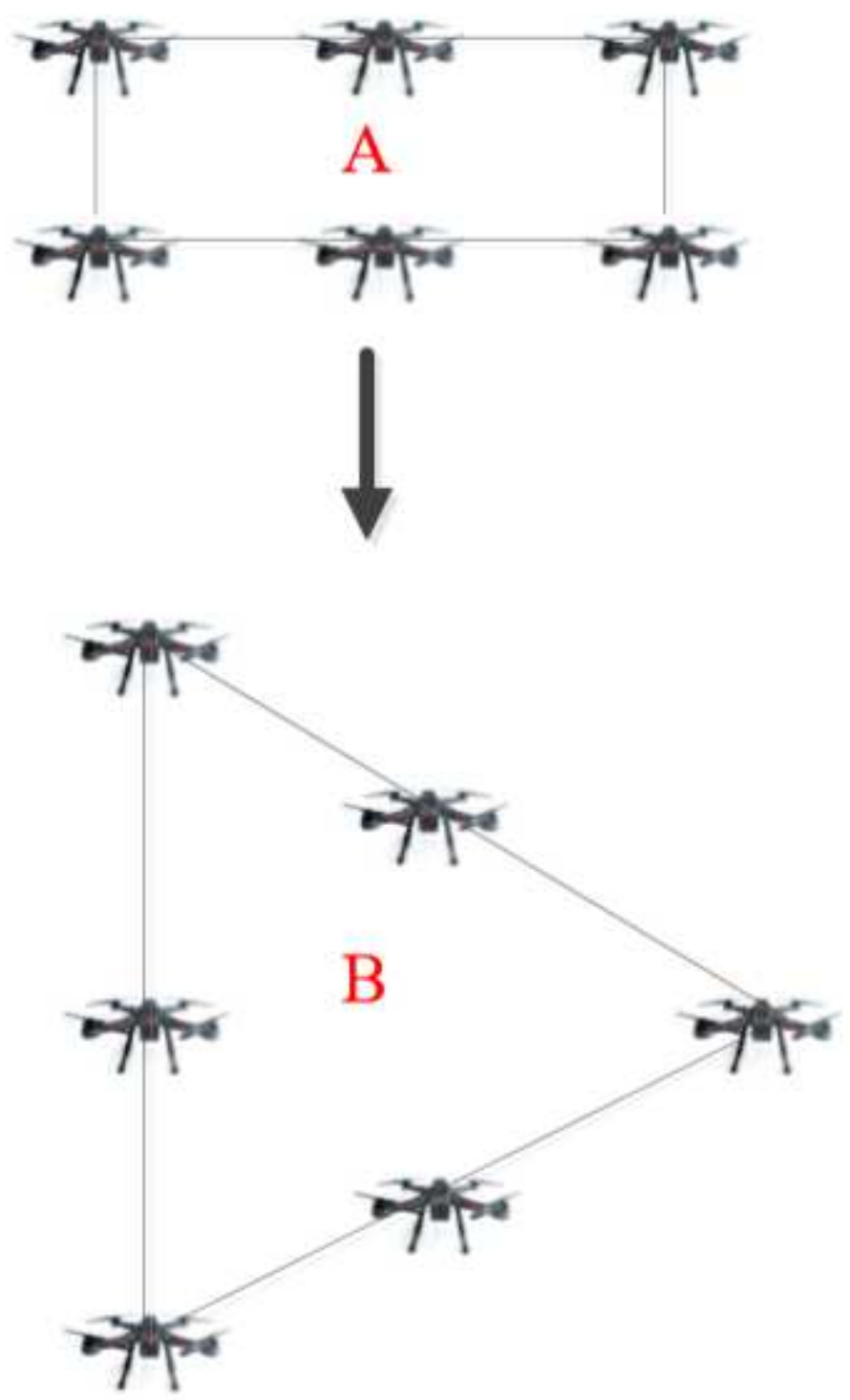

Figure 3

Formation transformation 


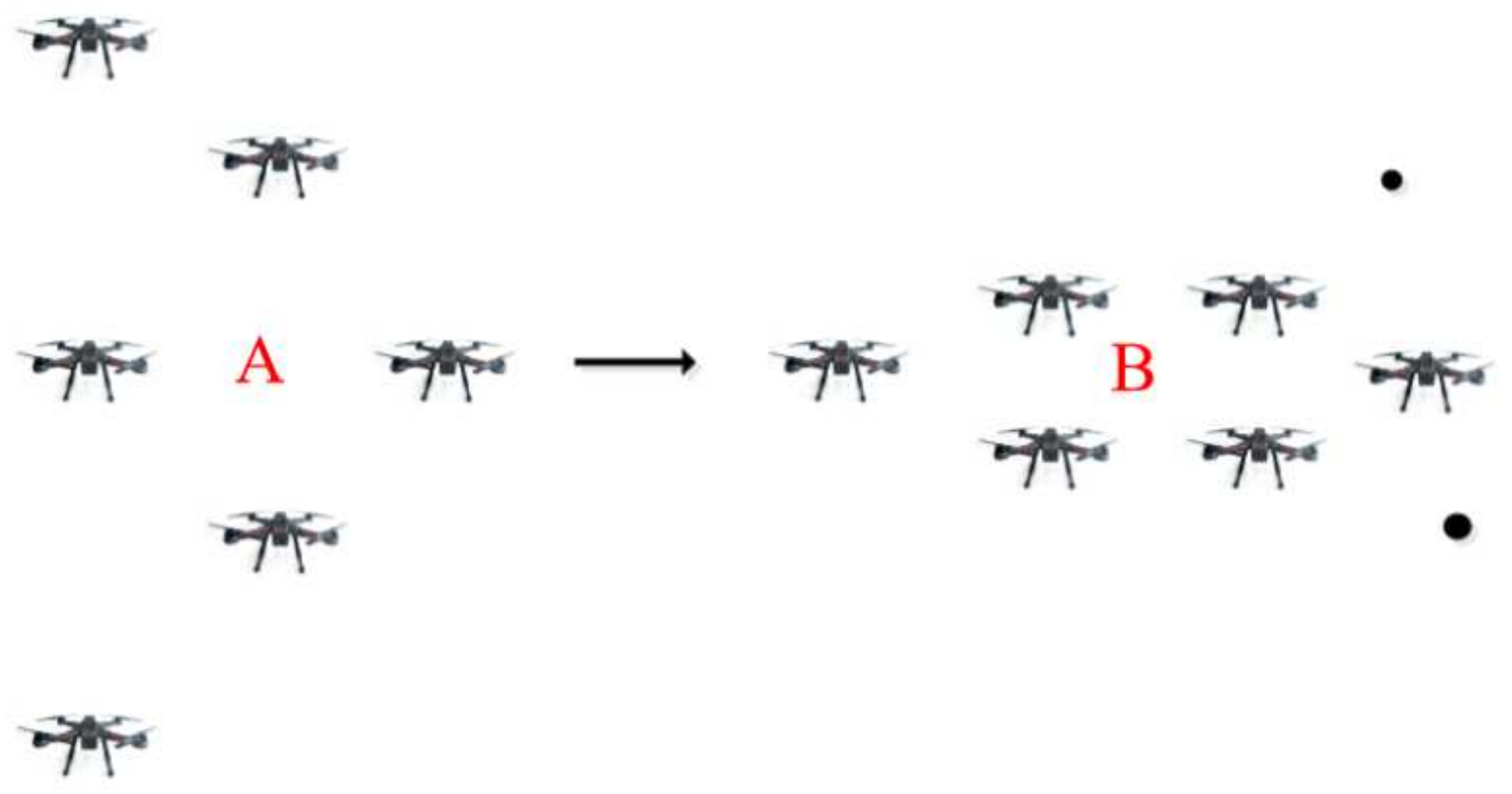

\section{Figure 4}

Reduction diagram of formation

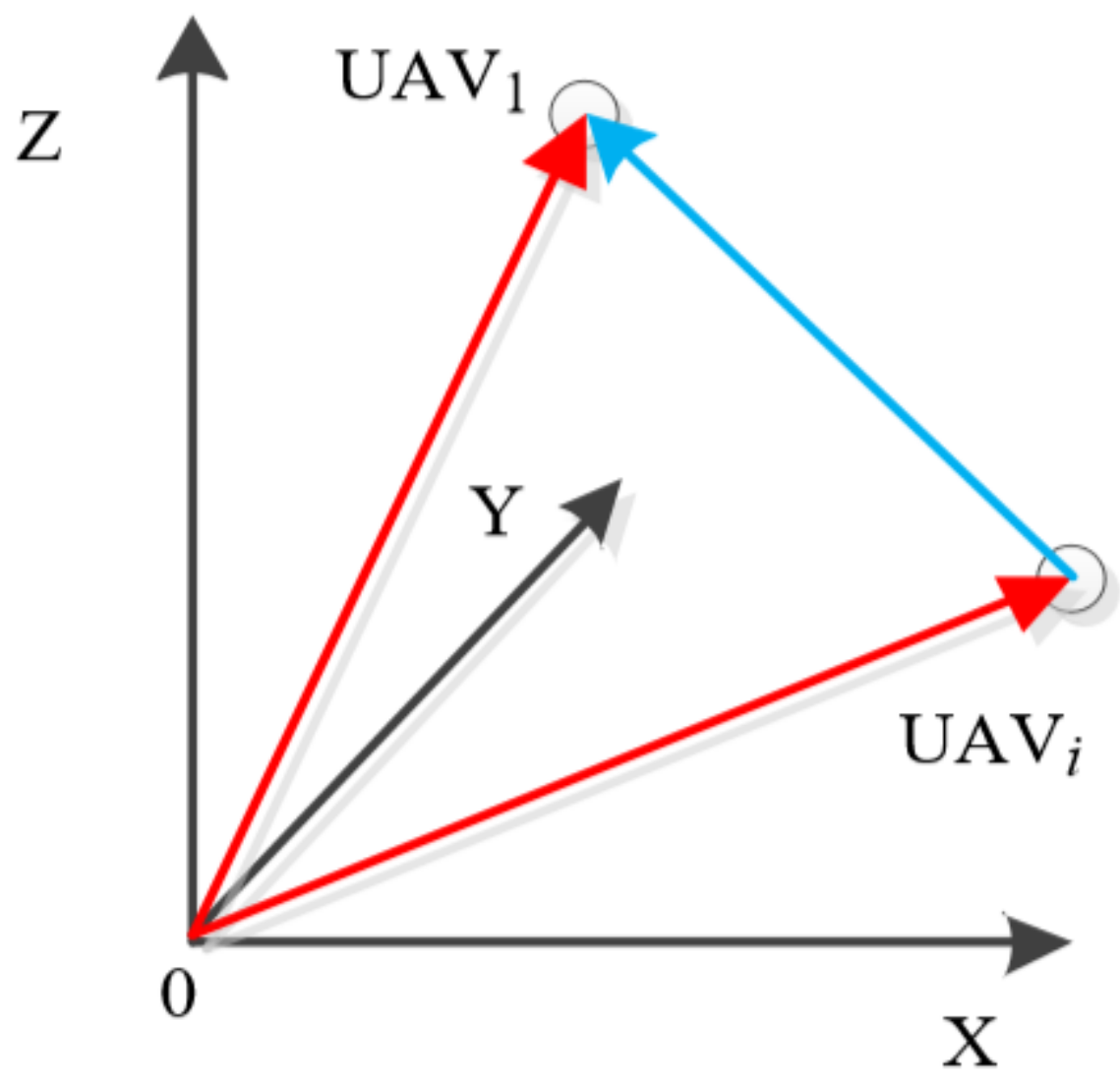


Figure 5

Relative position in formation between followed UAV and leader UAV

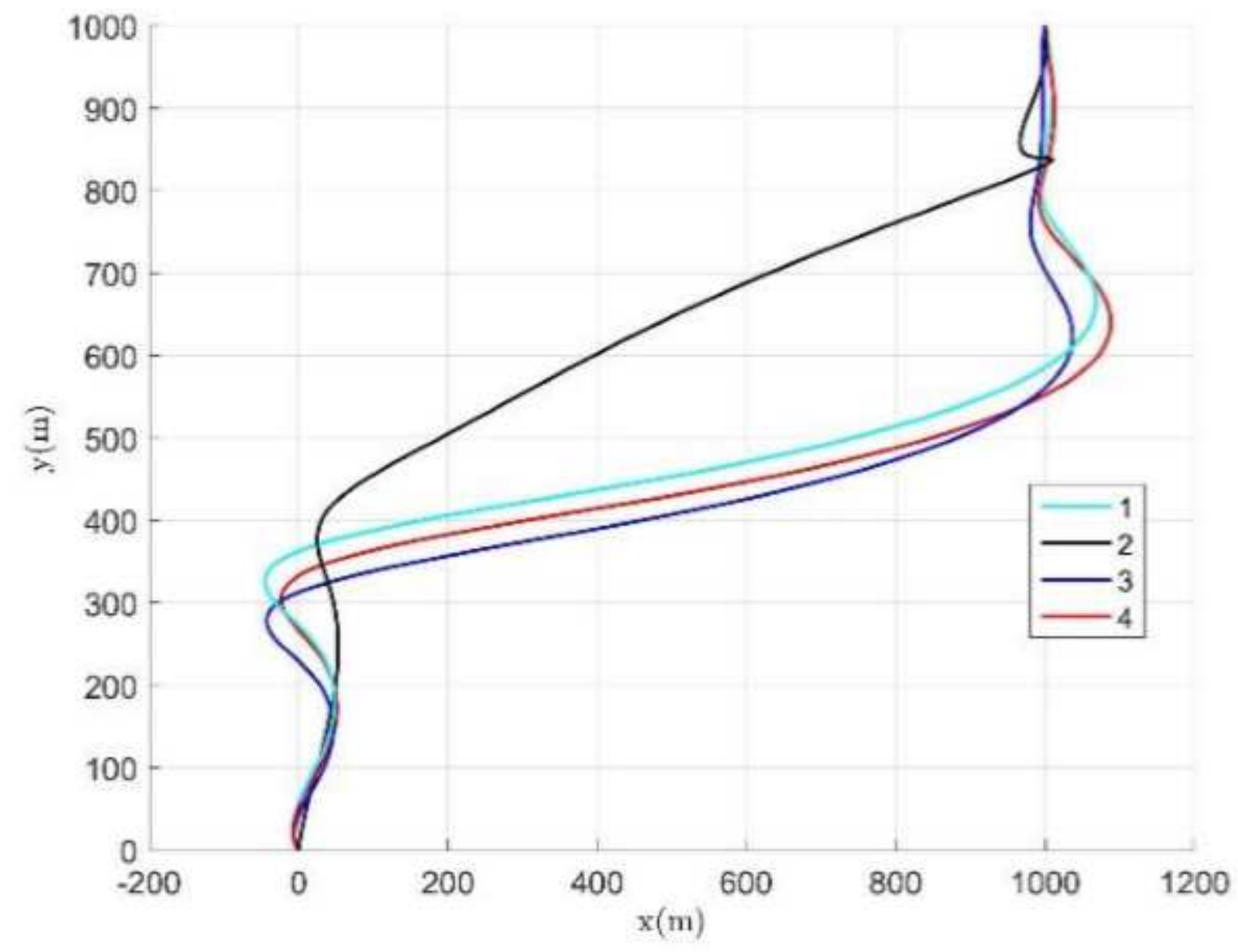

Figure 6

$x-y$ plane flight diagram 


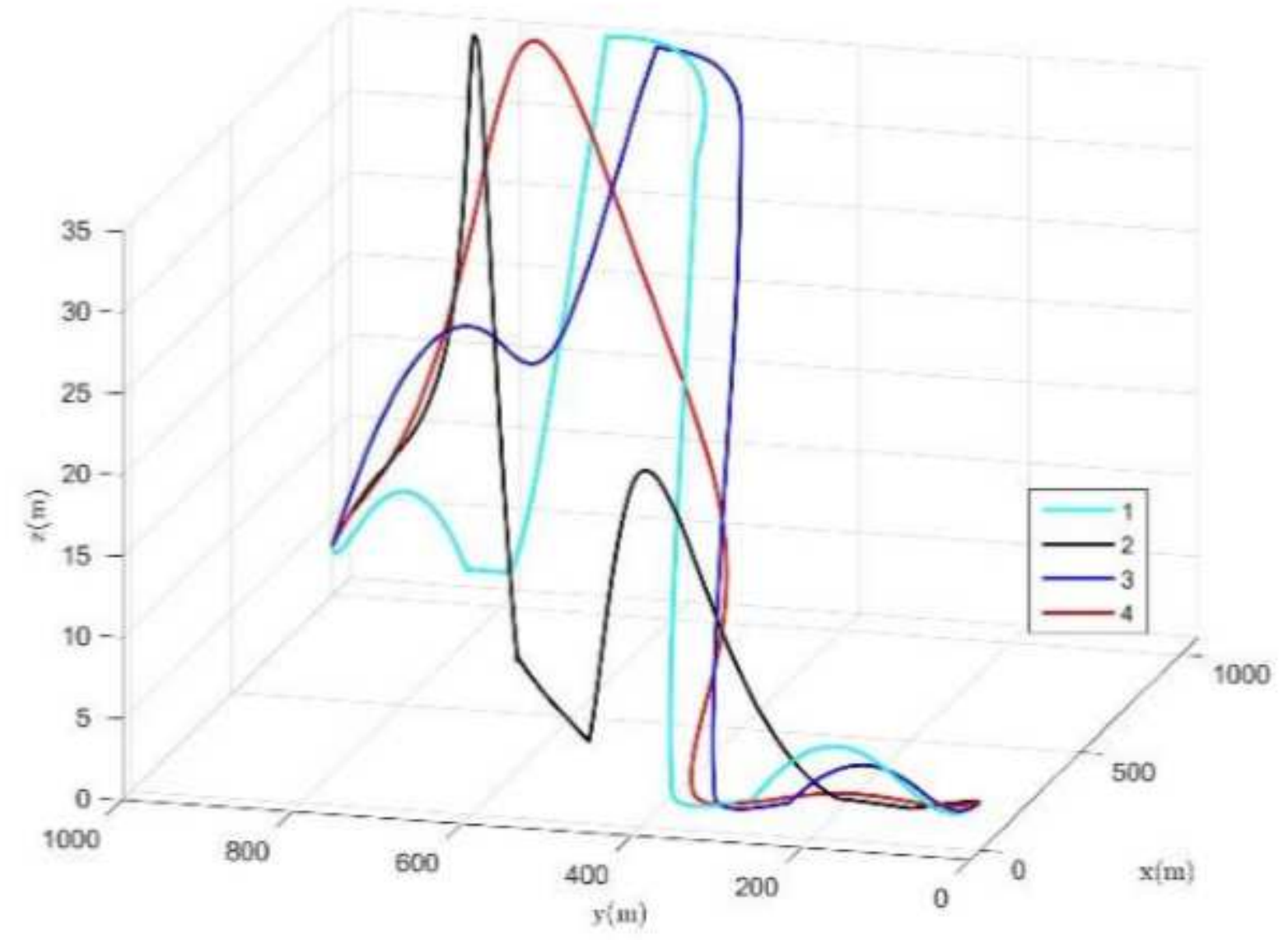

Figure 7

3D flight diagram 


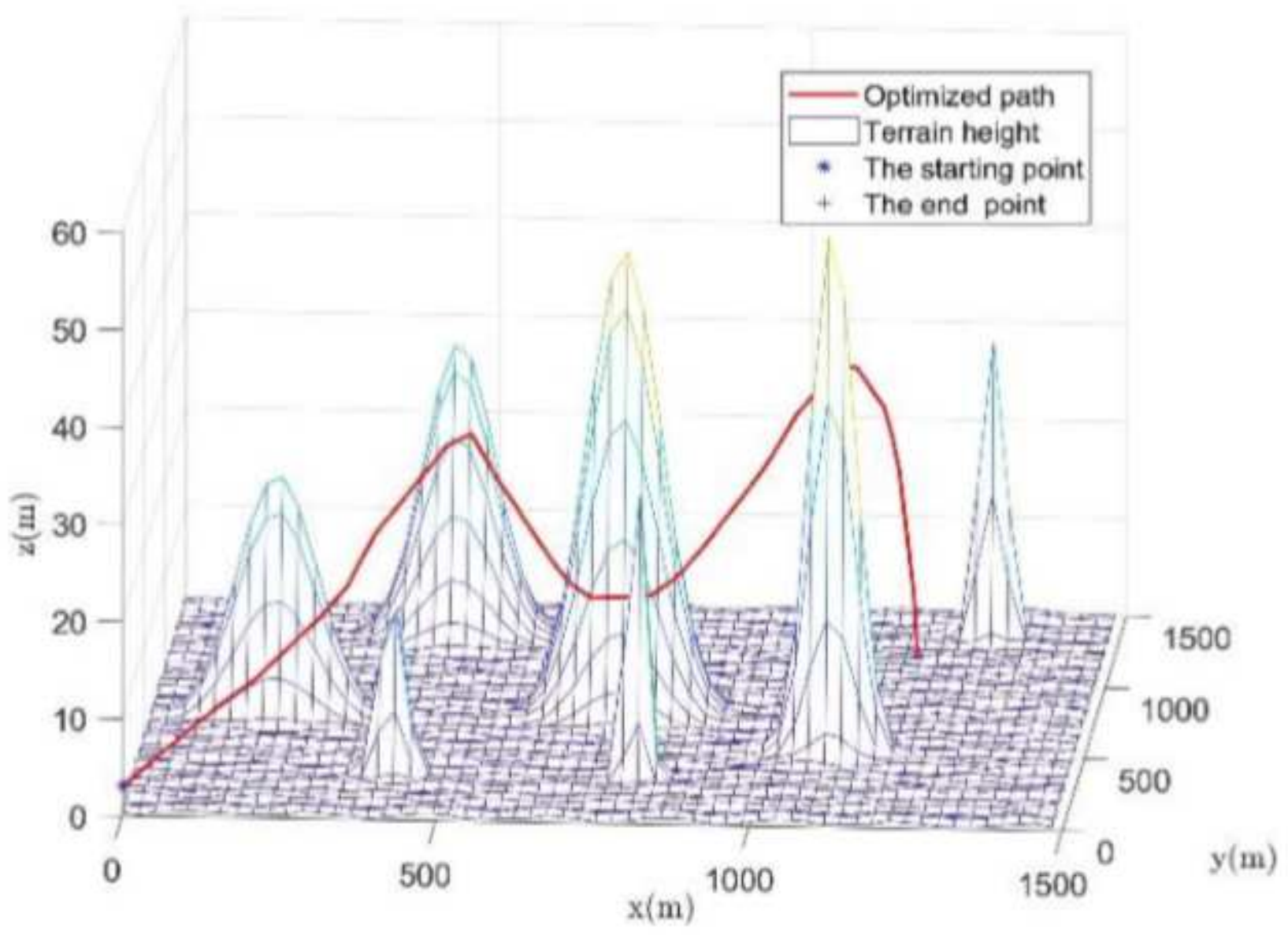

Figure 8

$3 \mathrm{D}$ view of the optimized path 


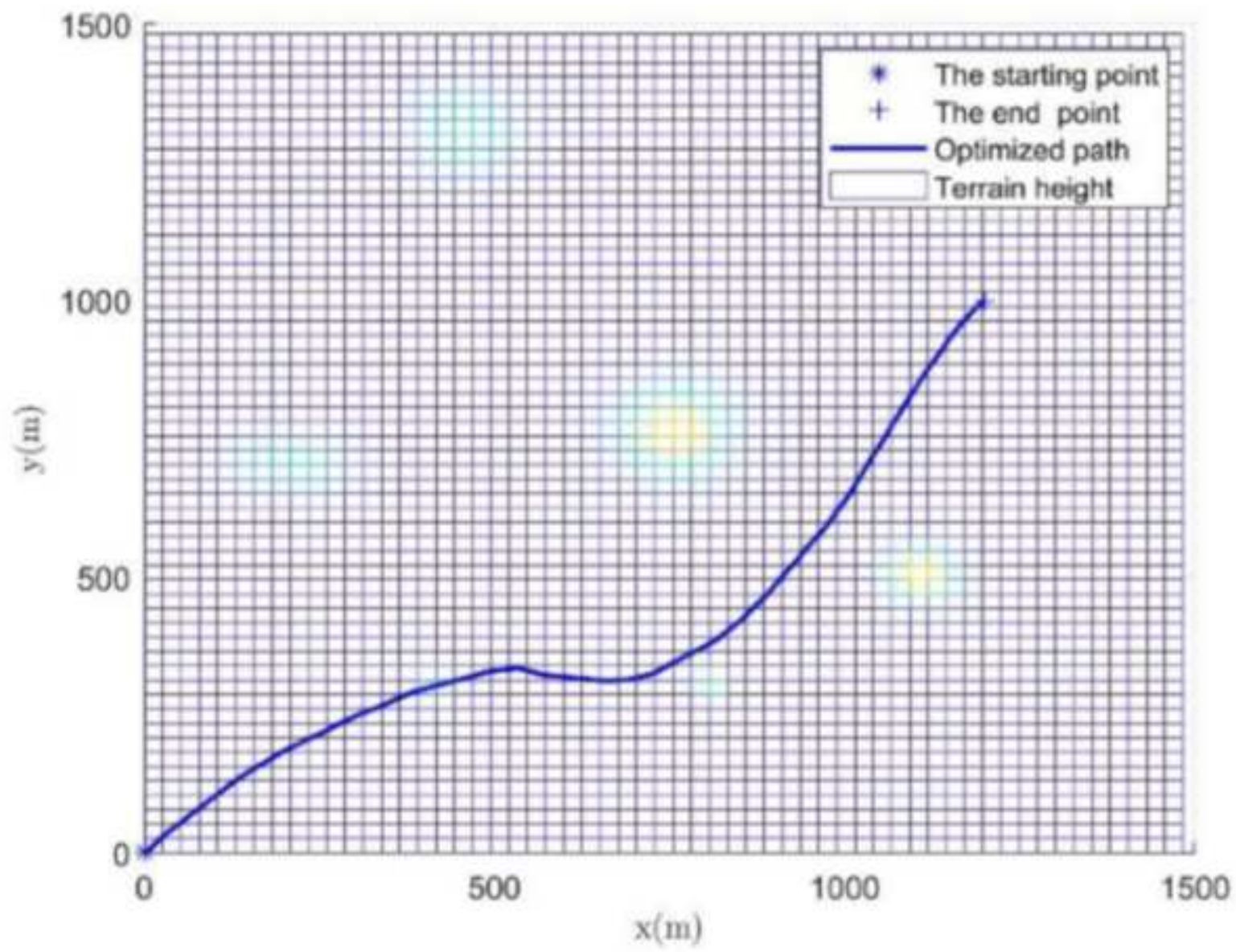

Figure 9

$x-y$ plane of the optimized path 


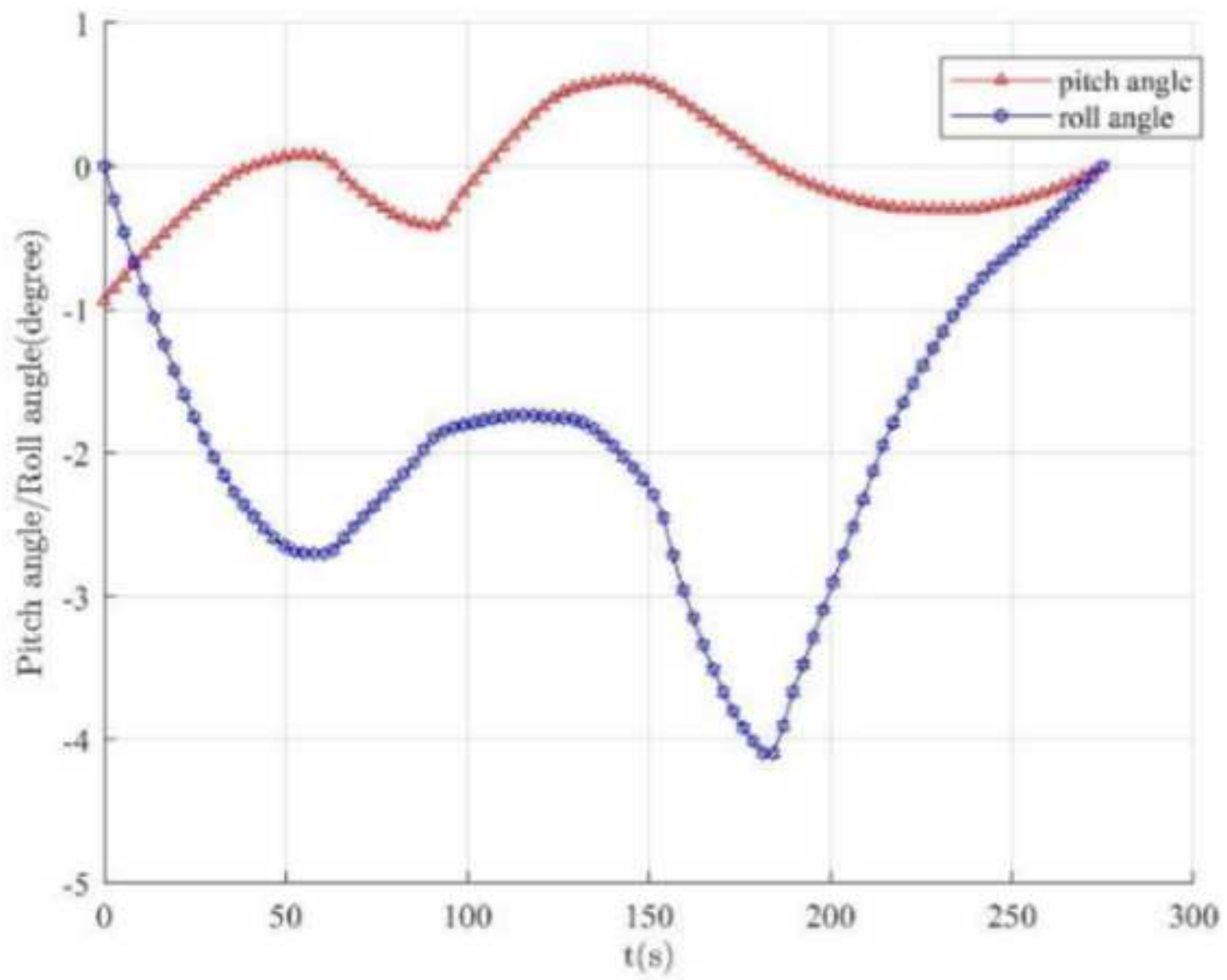

Figure 10

The curves of pitch angle and roll angle of the leader UAV 


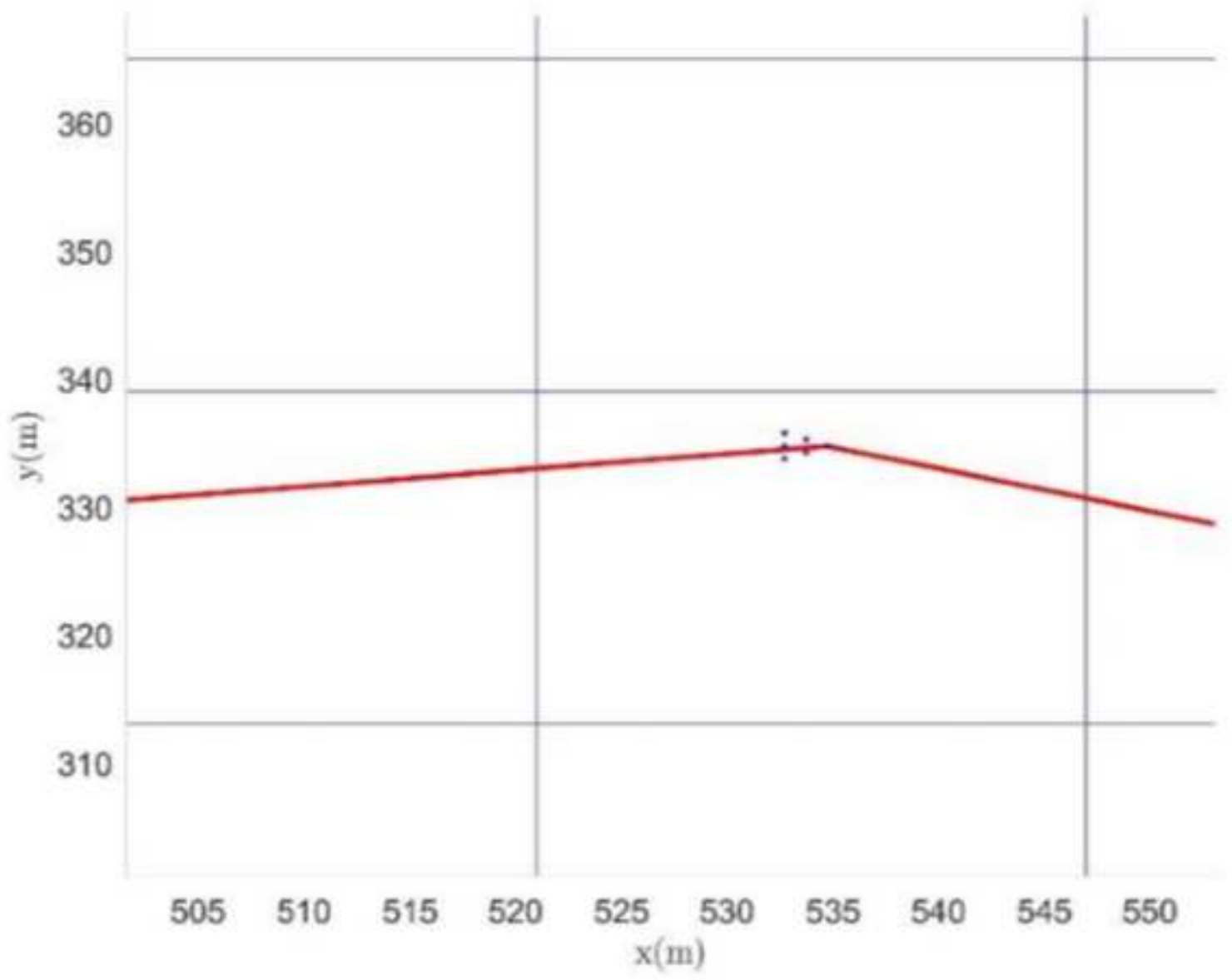

Figure 11

Formation of UAVs 


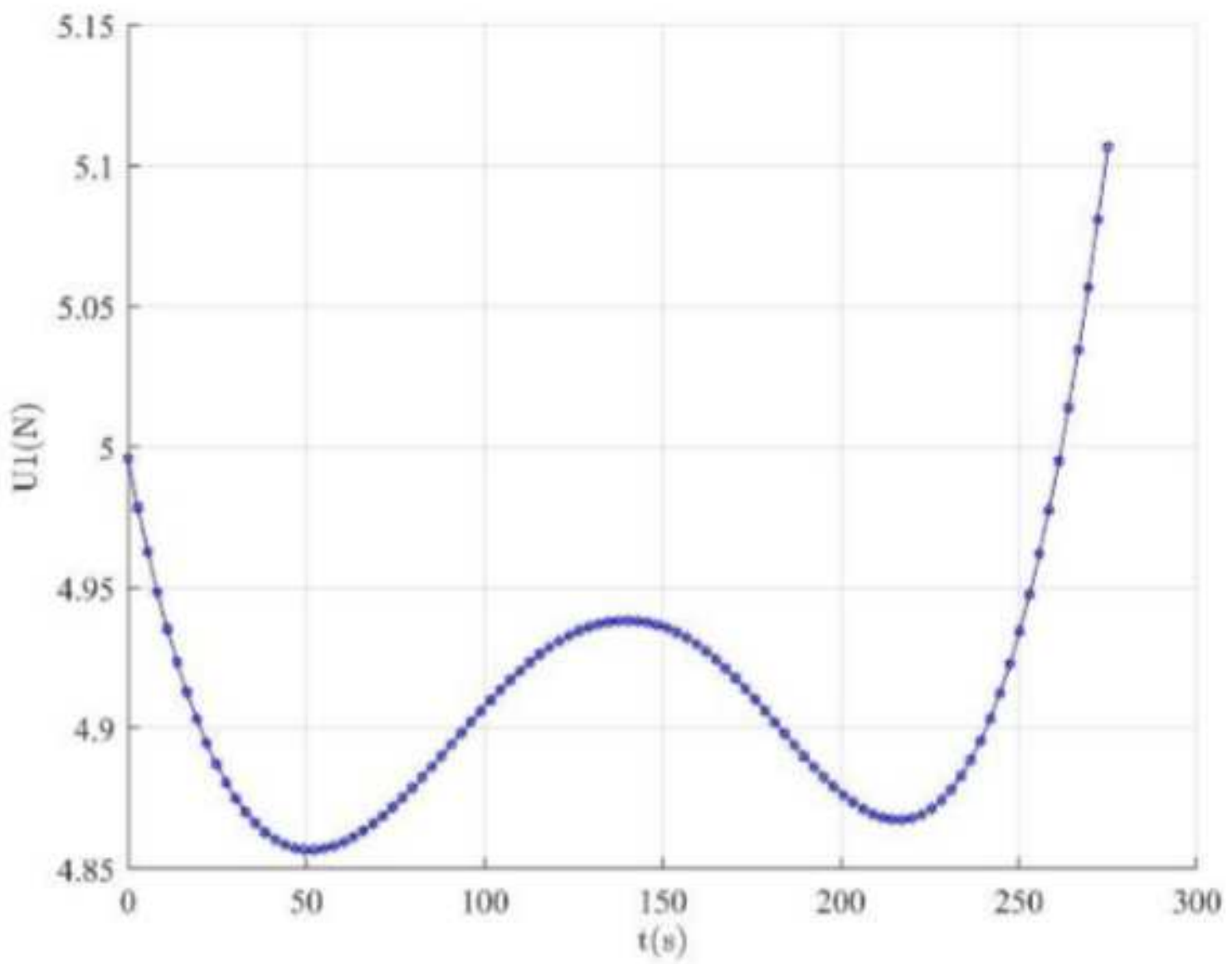

Figure 12

The curves of U1 of the leader UAV 


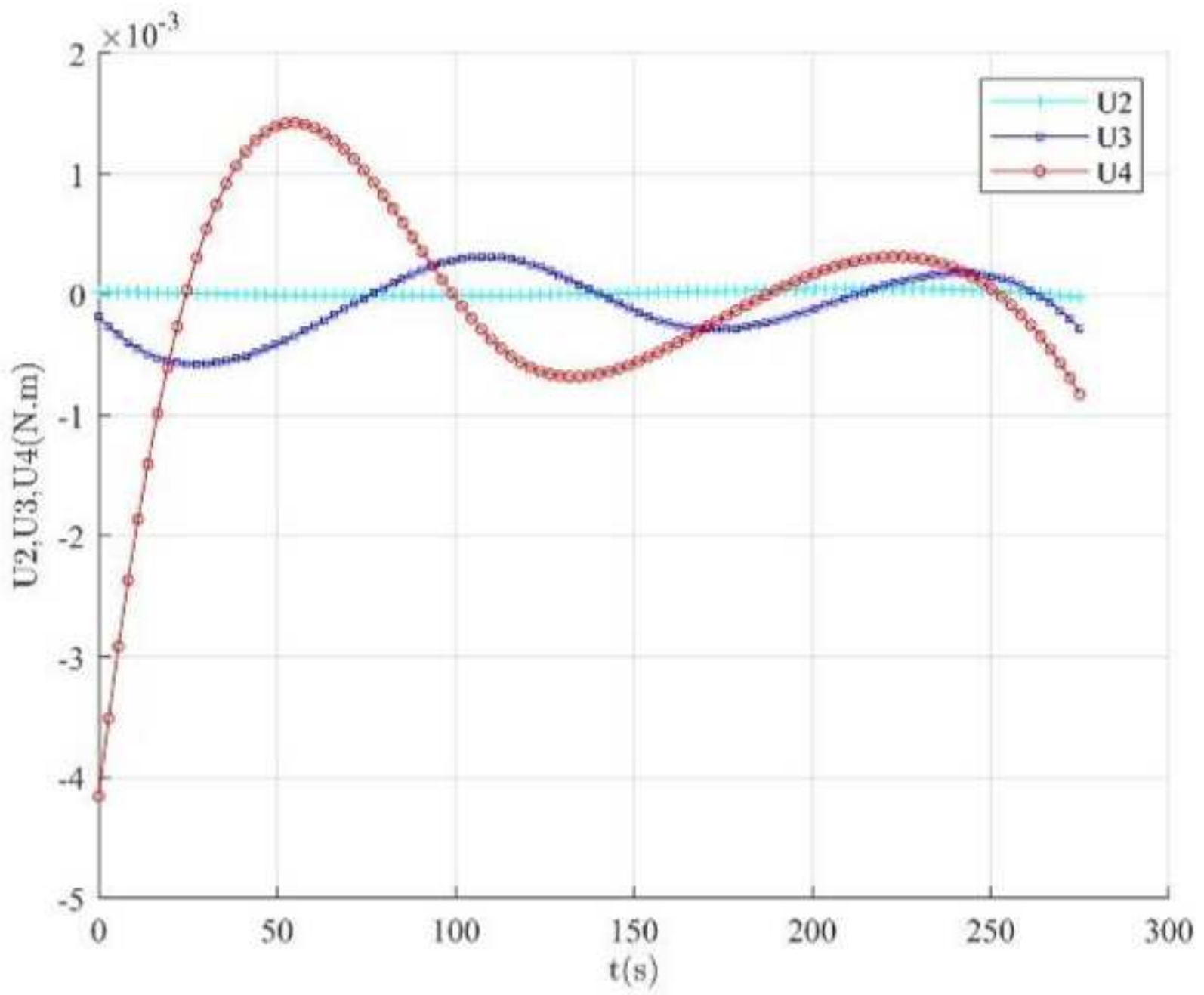

Figure 13

The curves of U2, U3 and U4 of the leader UAV 


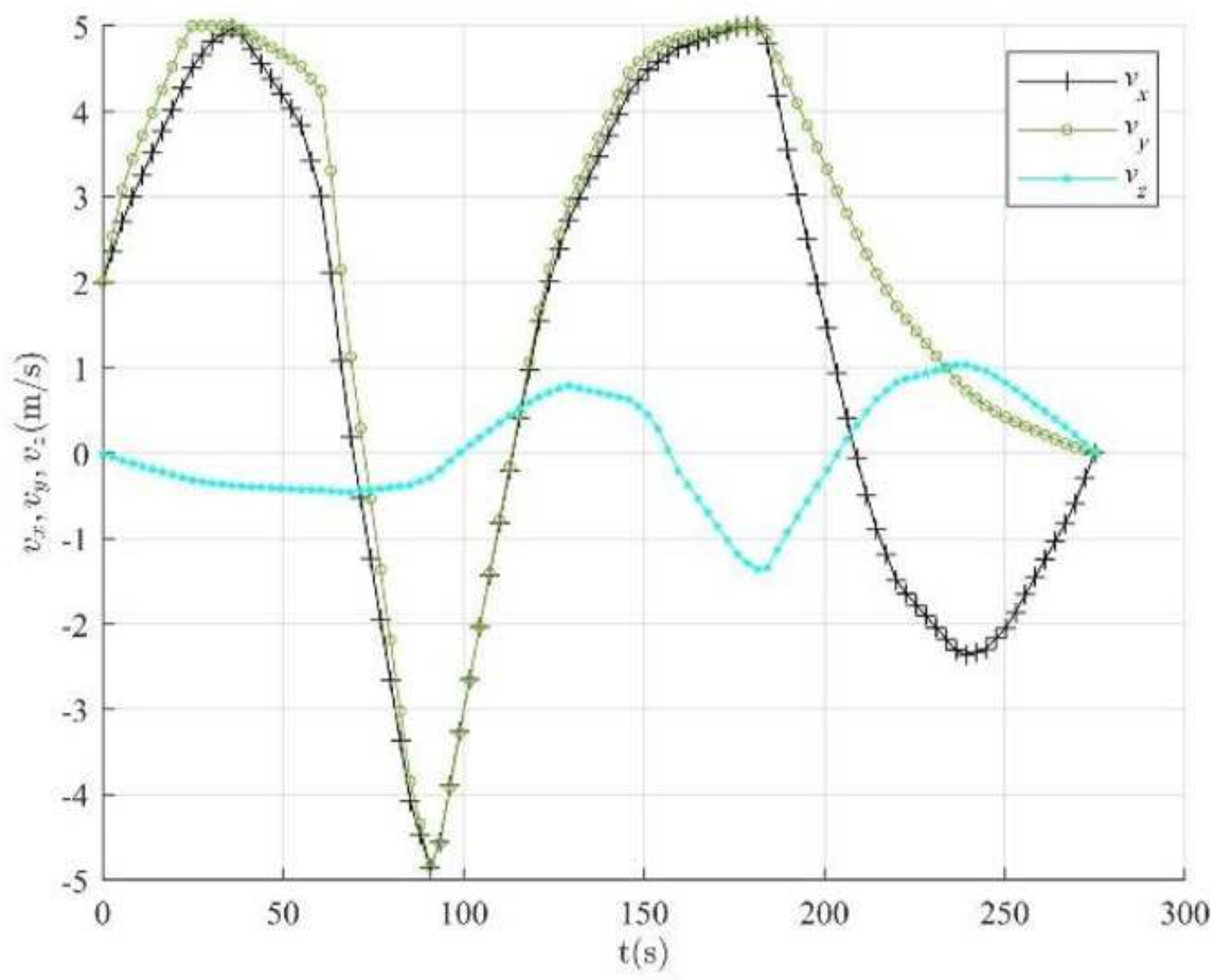

Figure 14

The curves of $v x$, vy and vz of the leader UAV 


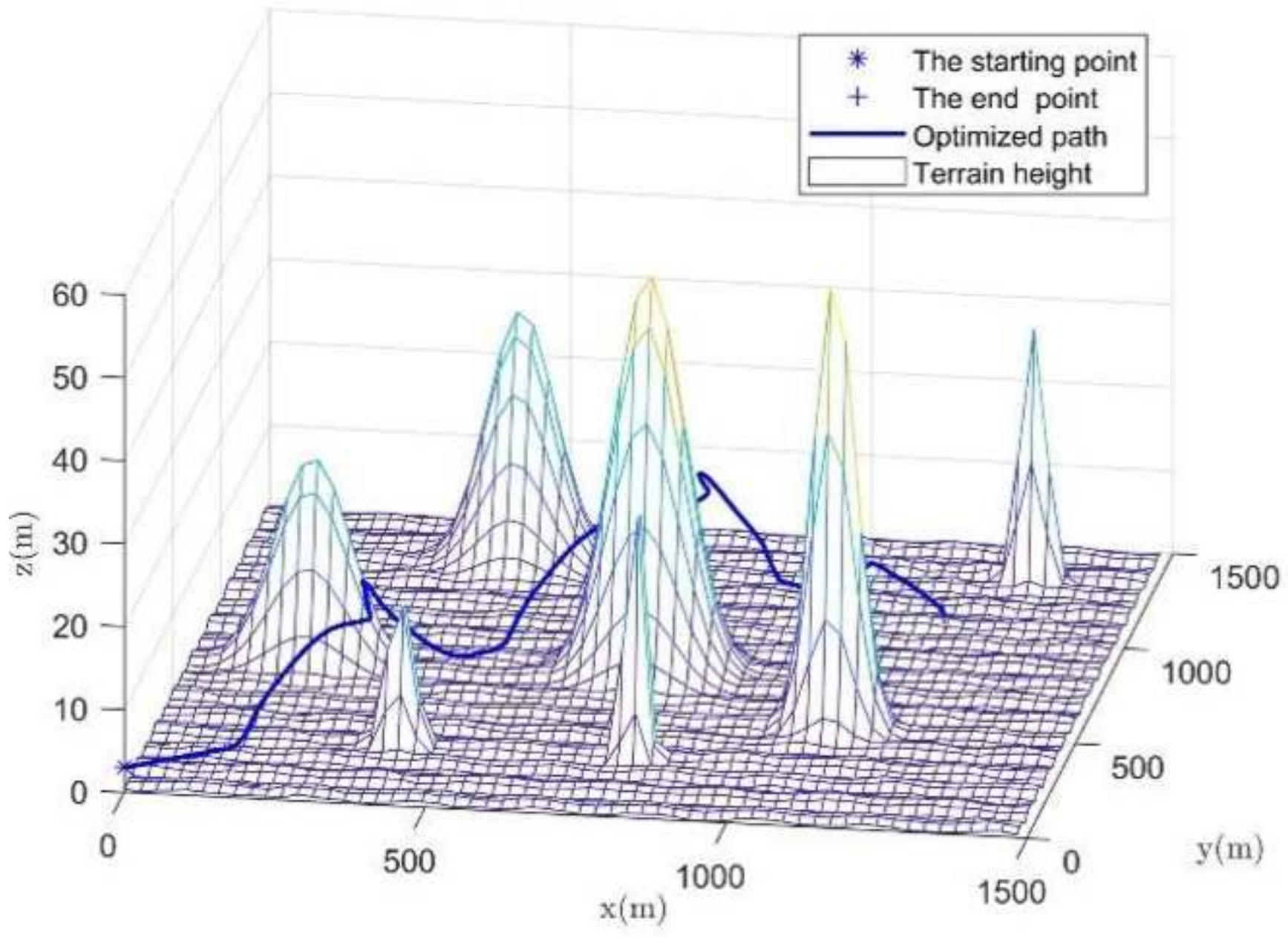

Figure 15

$3 D$ view of the optimized path 


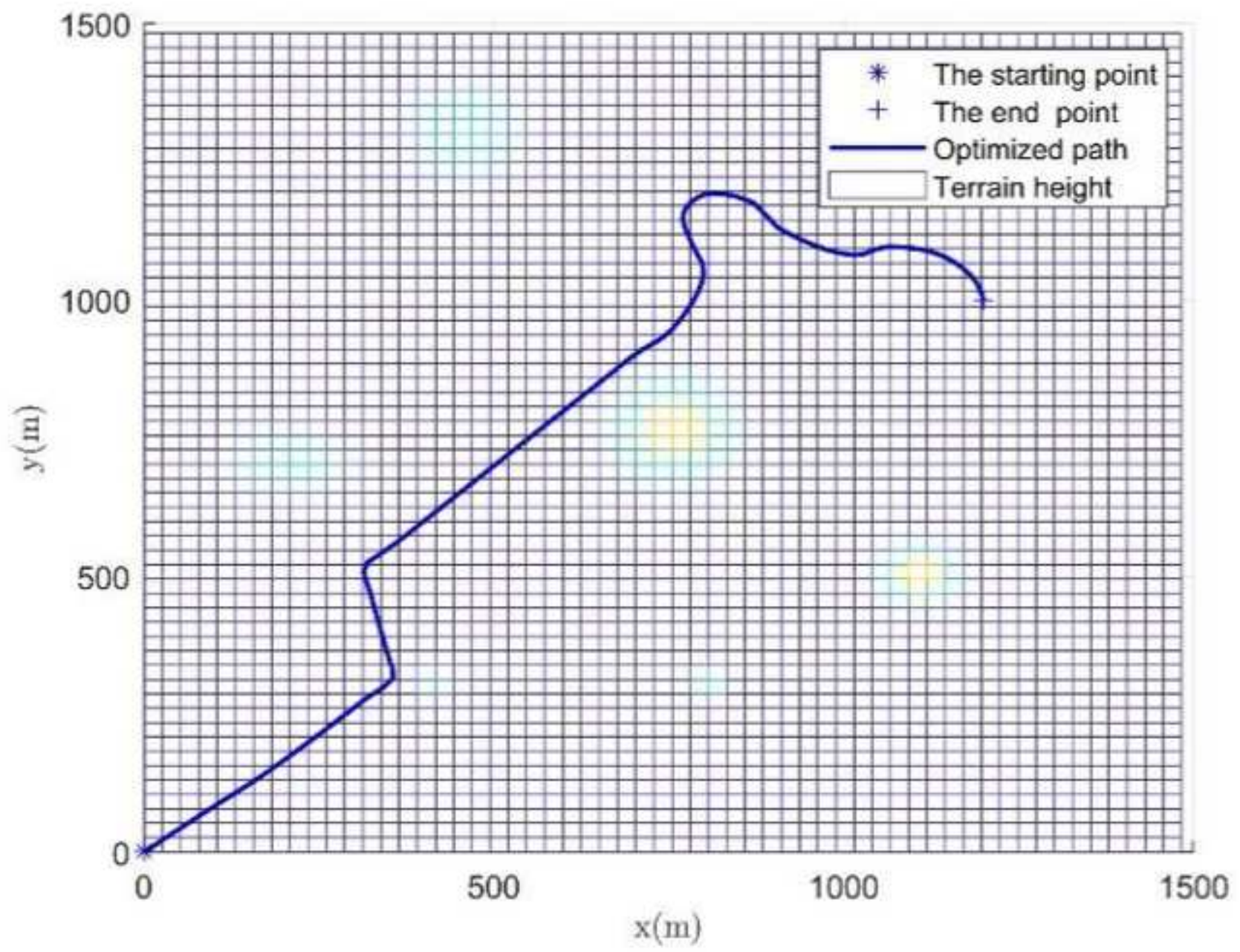

Figure 16

$x-y$ plane of the optimized path 


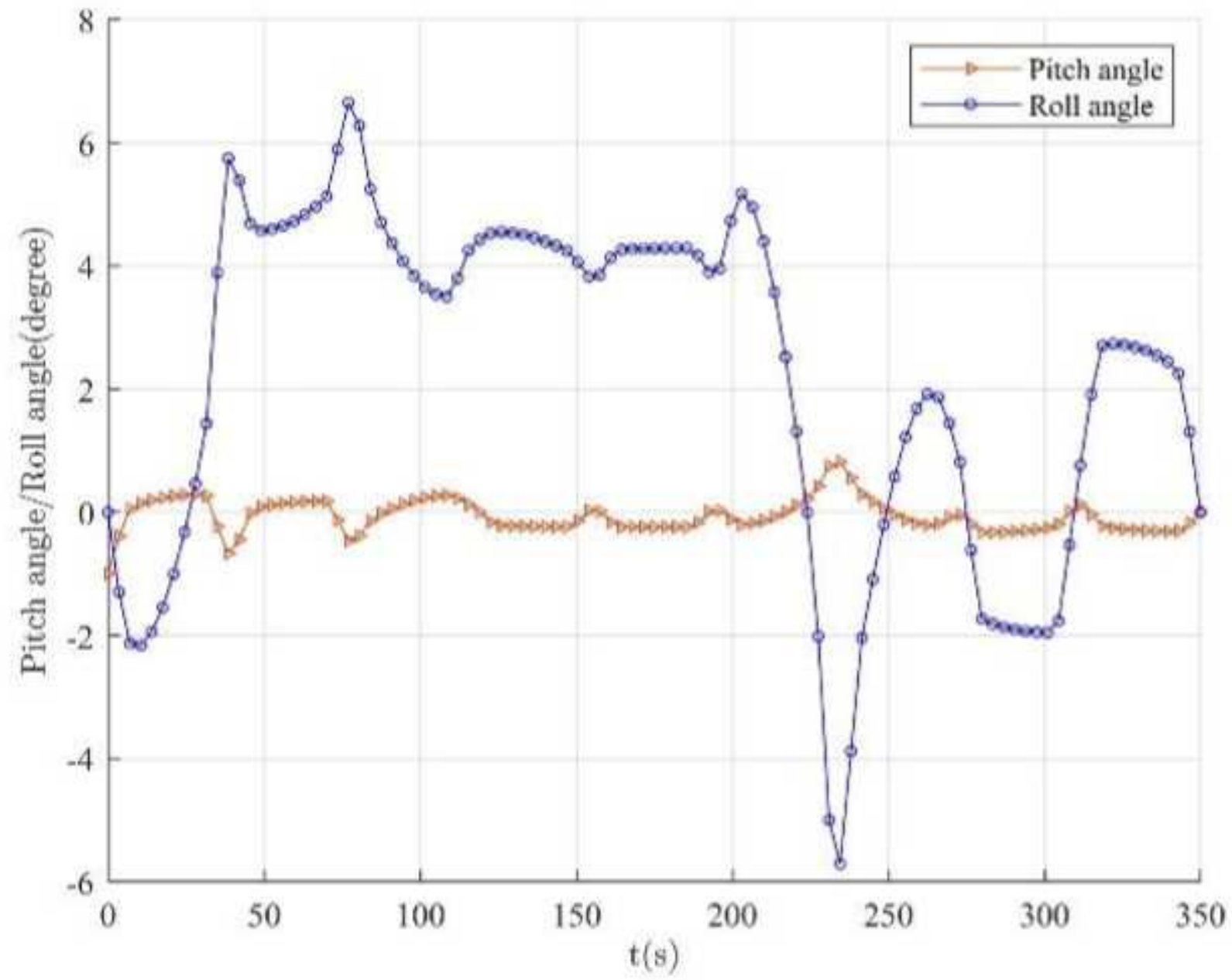

Figure 17

The curves of pitch angle and roll angle 


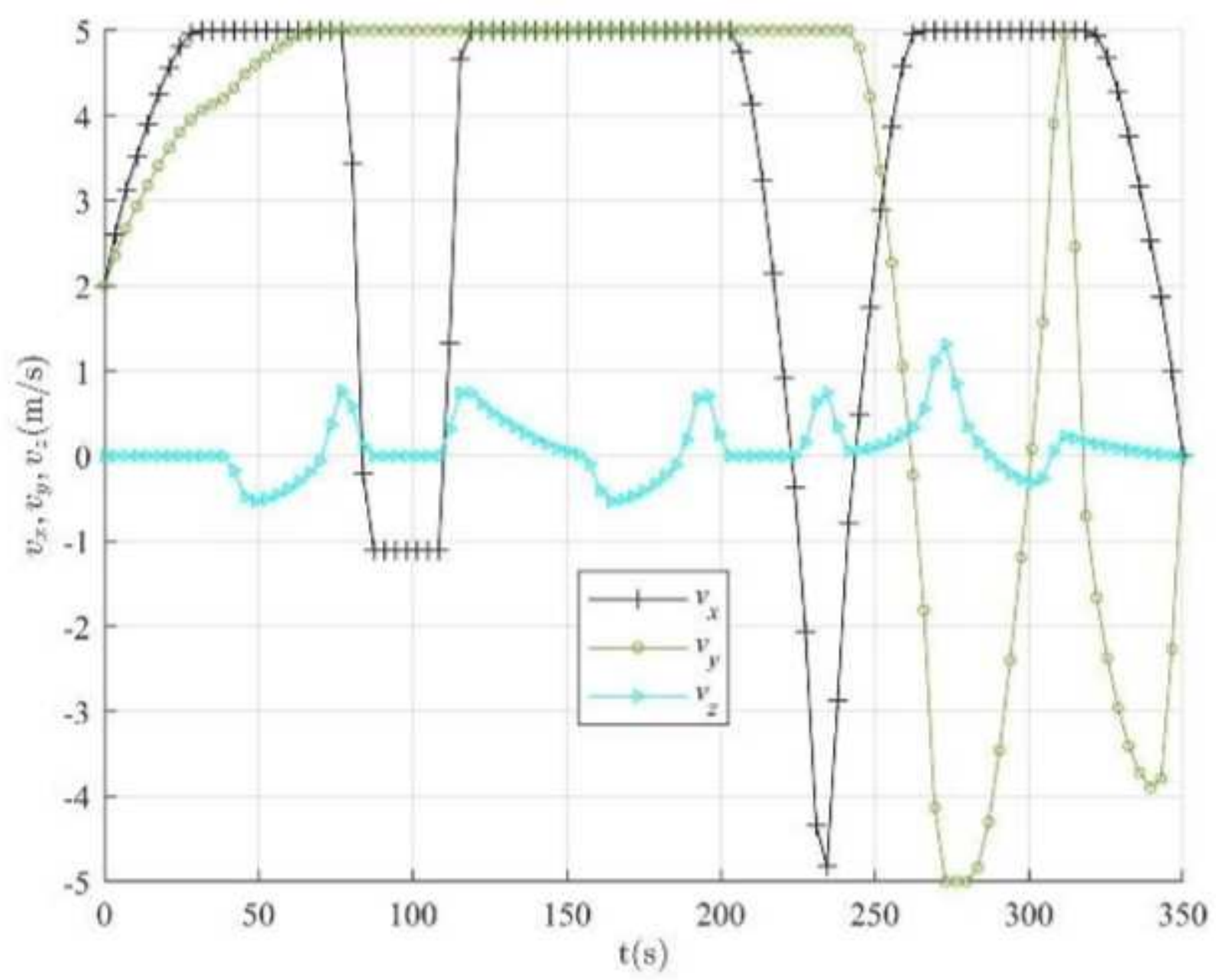

Figure 18

The curves of vx, vy and vz of the leader UAV of the leader UAV 


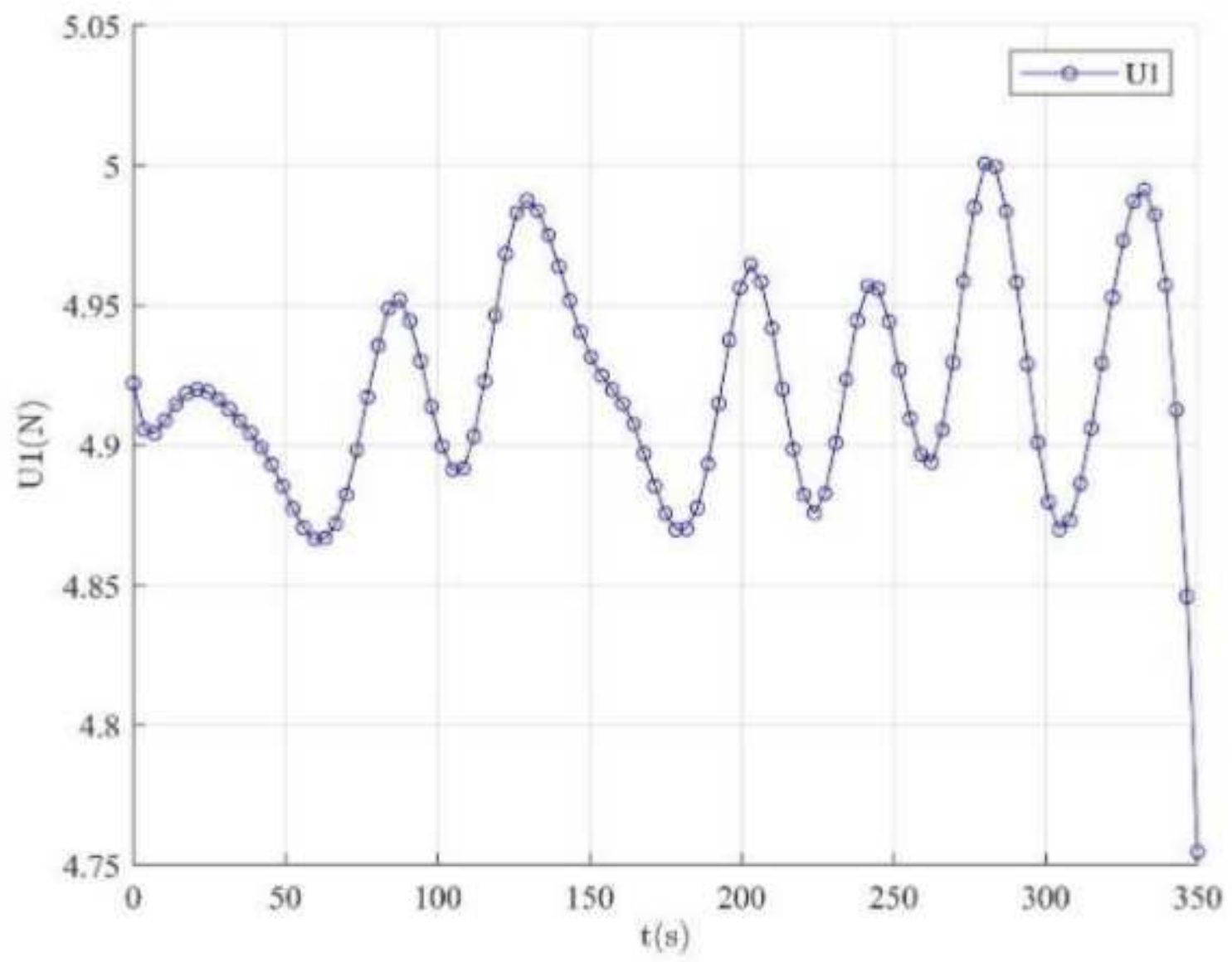

Figure 19

The curves of U1 of the leader UAV 


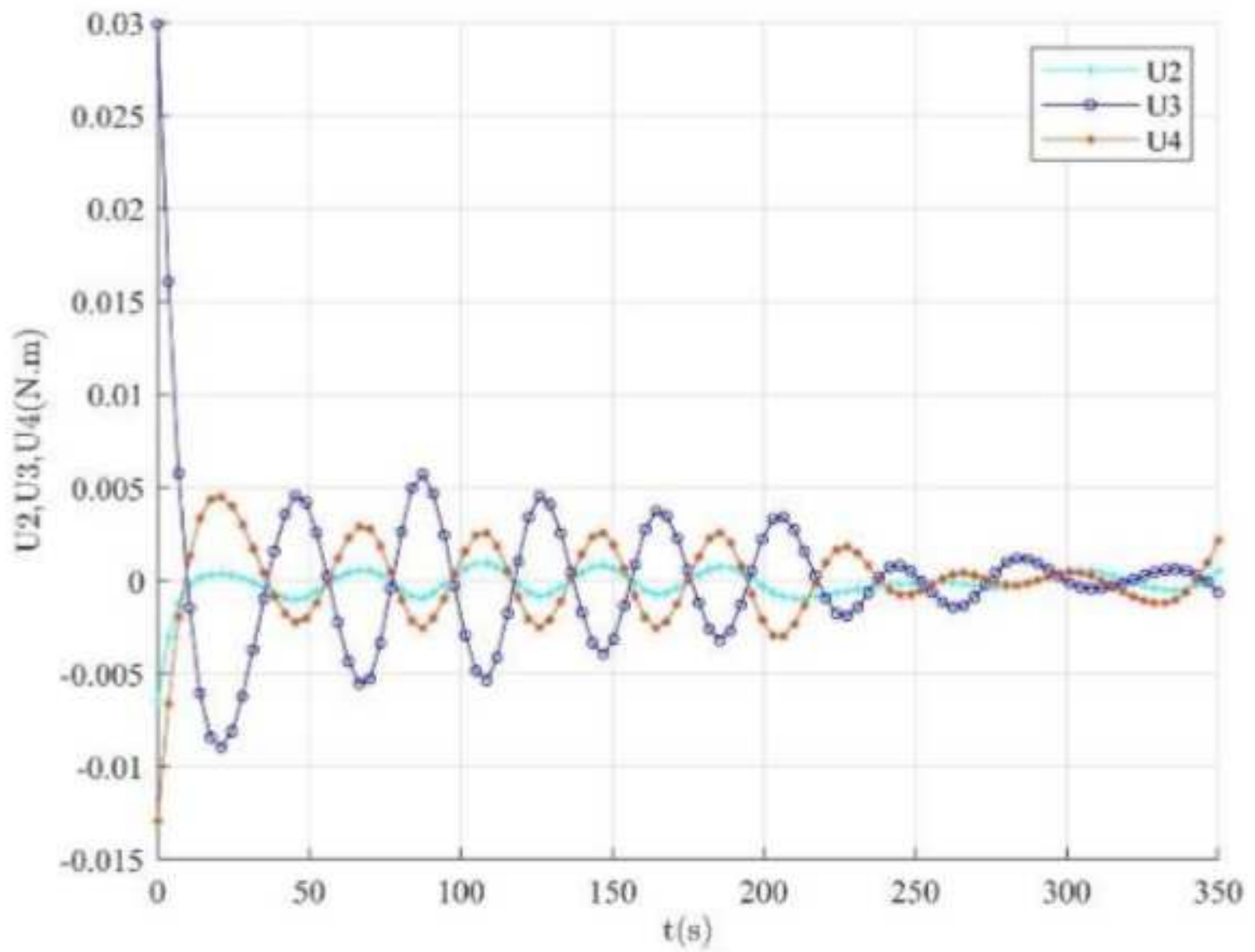

Figure 20

The curves of U2, U3 and U4 of the leader UAV 